\title{
A REVIEW OF CHIRONOMIDAE STUDIES IN LENTIC SYSTEMS IN THE STATE OF SÃO PAULO, BRAZIL
}

Fábio de Oliveira Roque; Leny Célia da Silva Correia;Susana Trivinho-Strixino; Giovanni Strixino.

Biota Neotropica v4(n2) http://www.biotaneotropica.org.br/v4n2/pt/abstract?article+BN03104022004

\author{
Date Received 05/20/2003 \\ Revised 07/19/2003 \\ Accepted 08/01/2003
}
Laboratório de Entomologia Aquática - Depto. de Hidrobiologia - Universidade Federal de São Carlos Washington Luis, Km 235. Cx. Postal 676. Fone: 16260-8316. Fax: 260-8310
E-mails: pfor@iris.ufscar.br.; plenycor@hotmail.com; strixino@power.ufscar.br

\begin{abstract}
Information on the chironomid fauna in lentic habitats in the State of São Paulo is gathered from the literature, and suggestions for future studies are provided.
\end{abstract}

Key words: Chironomidae, lentic systems, Neotropics, references

\section{Resumo}

Este trabalho reúne dados de estudos ecológicos sobre Chironomidae em ambientes lênticos do estado de São Paulo e propõe algumas sugestões para futuros estudos.

Palavras-chave:Chironomidae, sistemas lênticos, Região Neotropical, referências.

http://www.biotaneotropica.org.br 


\section{Introduction}

The Chironomidae is a species rich and abundant family of aquatic Diptera. They have a Cosmopolitan distribution, with a global number of species estimated at 8,000 to 20,000 (Coffman 1995). The family generally plays an important role in ecological studies (Armitage et al. 1995), environmental assessment (Rosenberg 1992), palaeoecological research (Walker 1995), agricultural entomology (Ferrarese 1993), and chironomids can also be important for public health (Cranston 1995).

In Brazil the first observations on chironomids were made as early as in 1805 . However, modern chironomid research began with the studies by Dr. Sebastião de Oliveira in the 1940ths (Messias 2000). Then, in the 1960th Dr. Ernst Josef Fittkau started working on the chironomid fauna in the Amazonian. The project was a cooperation between the Instituto Nacional de Pesquisas da Amazônia (INPA) and the Max-Planck-Institute for Limnology. Fittkau (2001) provides an overview of Chironomidae research in Brazil, with emphasis on the research in the Amazonian.

In the State of São Paulo, chironomid research was initiated in 1971 with the study on the benthic ecology of the Americana Reservoir carried out by Dr. Giovanni Strixino. Later, several projects have been developed with the purpose to increase the knowledge of the ecology of chironomids in reservoirs. This interest is related to the need of assessing the degree of and the impact of reservoirs on the main rivers in the State of São Paulo, and larvae of Chironomidae might be used in such impacts studies.

Unfortunately, taxonomic studies are scarce, as can be seen from the Catalog and Bibliography of Neotropical and Mexican Chironomidae (Spies \& Reiss 1996), the Chironomidae list of the State of São Paulo (Trivinho-Strixino \& Strixino 1999), and the "Brazilian chironomid page". Furthermore, except for the Chironomidae larvae identification manual (Trivinho-Strixino \& Strixino 1995) there is no practical manual to identify this fauna in Brazil.

Several factors have encouraged us to gather information about Chironomidae studies in lentic habitats in São Paulo: A) the BIOTA-FAPESP Program, chiefly the project "Inventory and Biology of freshwater Crustaceans, Insects and Mollusks of the State of São Paulo", which aims at integrating researchers and students from different laboratories and institutions to study taxonomical and ecological aspects of freshwater macroinvertebrates in the State of São Paulo; B) the small number of references to studies in the state of São Paulo cited in Brazilian and international articles; C) the necessity to establish a baseline to support future studies; and D) the necessity to provide a list of Chironomidae taxa, as well as a complete bibliography, in order to facilitate the exchange of information among researchers.

\section{Methodology}

We have tried to list all the published works on the ecology and taxonomy of Chironomidae in lentic habitats in the São Paulo State. Most references were obtained from Dr. Susana Trivinho Strixino's and Dr. Giovanni Strixino's archives, from the libraries of São Paulo universities, from personal contact with authors, and from internet databases. Abstracts from symposia, congresses and other meetings were not considered because they do not include comprehensive species lists and most of their results are published in articles. Identification level and morphospecies are cited here as they are written in the original references. When possible, we have added additional information about the environmental conditions at the sampling sites not given by the authors. We emphasize that we consider correcting misidentifications and nomenclatural changes to be outside the scope of this article.

\section{Results and comments}

A total of 60 studies were found (Table 1), including 31 scientific articles, $7 \mathrm{PhD}$ theses, 18 master- and lowerlevel theses, and 4 reports. In addition, 5 personal communications and works in preparation were included. The majority of the studies are from small reservoirs in the São Carlos district. Reservatório do Monjolinho (São Carlos) is the most intensively studied reservoir, being the focus of 10 articles.

A total of 276 taxa were listed in Table 2, of which only 36 are formally named species. It is important to point out that the number of taxa listed in Table 2 is probably overestimated, because a given morphospecies may be listed as "species A" in a study and "species B" in another study. Chironomus, Ablabesmyia, Polypedilum and Tanytarsus are the genera most frequently recorded from the reservoirs. It is important to note that these genera are very species rich in the Neotropical region and undoubtedly comprise many undescribed species.

Some taxa listed in Table 2 (e.g. Ablabesmyia annulata, Kiefferulus, and Zavrelia) are not recorded from the Neotropical region by Spies \& Reiss (1996), indicating possible misidentifications. For others further studies of the material carried out at the Laboratório de Entomologia Aquática (UFSCar) have led to the following name changes (previous name $\rightarrow$ actual name): Micropsectra sp. 1 (Albuquerque 1990) $\rightarrow$ Tanytarsini Gênero C (Trivinho-Strixino \& Strixino 1995); Micropsectra sp. 2 (Albuquerque 1990) $\rightarrow$ Tanytarsini Gênero B (Trivinho-Strixino \& Strixino 1995); Microtendipes sp. 2 (Albuquerque 1990) $\rightarrow$ Beardius sp. 3 (Trivinho-Strixino \& Strixino 1995) $\rightarrow$ Beardius parcus Reiss $\&$ Sublette, 1985; Nimbocera sp. 2 (Albuquerque 1990) $\rightarrow$ Nimbocera paulensis Trivinho-Strixino \& Strixino, $1991 \rightarrow$ Caladomyia ortoni Säwedal, 1981 (Trivinho-Strixino \& Strixino 2003); Paracladopelma sp.1 (Trivinho-Strixino \& 
Strixino 1993) $\rightarrow$ Harnischia sp.1 (Trivinho-Strixino \& Strixino 1995); Synendotendipes (Correia 1999) $\rightarrow$ Endotribelos; Tribelos sp. 1 (Trivinho-Strixino \& Strixino 1995) $\rightarrow$ Endotribelos; Cladotanytarsus (Trivinho-Strixino $\&$ Strixino 1995) $\rightarrow$ Caladomyia friederi Trivinho-Strixino \& Strixino, 2000; Gênero B (Trivinho-Strixino \& Strixino 1995) $\rightarrow$ Aedokritus coffeatus Trivinho- Strixino, 1997; Gênero A sp. 4 (Trivinho-Strixino \& Strixino 1995) $\rightarrow$ Fissimentum desiccatum Cranston \& Nolte, 1996; Gênero Asp. 2 (TrivinhoStrixino \& Strixino 1995) $\rightarrow$ Fissimentum sp. 2 Cranston \& Nolte, 1996; Pedionomus (Albuquerque 1990) $\rightarrow$ Polypedilum (Asheum); Asheum beckae Sublette, 1964 $\rightarrow$ Polypedilum (Asheum) beckae (Sublette, 1964); Nimbocera sp. 1 (Albuquerque 1990) $\rightarrow$ Nimbocera rhabdomantis Trivinho-Strixino \& Strixino, $1991 \rightarrow$ Tanytarsus rhabdomantis (Trivinho-Strixino \& Strixino, 1991), see Trivinho-Strixino \& Sanseverino (2003).

The high number of morphospecies shown in Table 2 highlights the deficiency of knowledge on Chironomidae taxonomy in the State of São Paulo. Most of the studies do not provide information on morphospecies, so that it is difficult to make comparisons with other works. In addition, there is no standardization of morphospecies in the majority of Chironomidae studies (Table 2), not even among those carried out in the same research group.

Although chironomid research has become increasingly popular in recent years, there are many challenges to overcome (see e.g. Epler, 2001). Recently, Lewinsohn \& Prado (2003) suggested several actions and priorities to improve the general knowledge of Brazilian biodiversity, which are also valid for Chironomidae. In the present study, we would like to focus on some particular aspects, which were intensively discussed at the latest Brazilian Chironomidae Symposiums:

\subsection{Cooperation}

- National and international cooperation among ecologists, taxonomists, biologists is fundamental to improve the knowledge of the Chironomidae in the Neotropical region, as pointed out by Fittkau (2001).

\subsection{Information organization}

- There is an urgent need for organizing and publishing the contents of private and public chironomids collections.

- Considering the expected increase in the taxonomic knowledge of the Brazilian chironomids in the coming years, we suggest that new identification guides is produced and updated continuously. The publication of electronic keys is strongly recommended, following the style of e.g., Epler (2001).

- Just like the type material of formally named taxa, voucher specimens of other species should also be deposited in well-maintained collections in Brazil. Prior to deposition or publication specialists should verify the identification of voucher specimens, and of any other specimens of special importance in a particular study. Taxonomists generally do this, while ecologists often do not. Establishing and/or improving such collections could be very helpful for nonspecialists.

\subsection{Methodological aspects}

- Agreeing with Fittkau (2003), high priority to taxonomic studies is recommended. Modern chironomid taxonomy aims at obtaining and integrating knowledge of all developmental stages. Therefore, the recommended procedure is rearing of larvae to obtain pupae and adults. Admittedly, this demands some effort and experience, but the advantages override these drawbacks. For example, each additional reliably associated life stage available for study multiplies the chances for correct identification and ecological interpretation of the species in question. Some methods for rearing chironomids were recently published by Mendes (2002); see also Epler (2001).

- It would be inappropriate here to recommend specific geographic areas for further studies, since the more intensively sampled areas still have their fauna poorly known.

- A minimum standard for environmental assessment procedures should be established that would allow comparisons between independent studies. This issue should be discussed at future meetings.

- Morphospecies concepts should also be standardized to allow comparative studies. We consider that, although descriptions of morphospecies do not lead to validity status equal to that of formally named taxa, they should be included in theses and dissertations in order to contribute to standardization and to future formal descriptions.

- When possible, the collection of larvae, pupae and adults is strongly recommended for any study involving chironomid richness. Particularly the collection of pupa exuviae does not require elaborated methodology or instruments, and adds only a few minutes to the field effort. This often also yields valuable specimens of drowned or emerging adults along with the cast skins. Some methods for collecting aquatic and terrestrial insects are described by New (1998).

Finally, the use of Chironomidae information in biomonitoring and biodiversity conservation programs has increased during the last decade. These applied approaches require that researchers from various fields cooperate and that results from different areas are considered when studying the multiple aspects related to the Chironomidae. 
Table 1. Summary of Chironomidae studies in lentic environments in the State of São Paulo, Brazil.

\begin{tabular}{|c|c|c|c|c|c|c|c|}
\hline $\begin{array}{c}\text { Entry } \\
\text { No. } \\
\mathrm{S}^{\mathrm{R}} \\
\end{array}$ & Site (S) & Reference (R) & $\begin{array}{l}\text { Geographic } \\
\text { coordinate }\end{array}$ & $\begin{array}{c}\text { Hydrographic } \\
\text { basin }\end{array}$ & $\begin{array}{l}\text { Area } \\
\text { (ha) } \\
\end{array}$ & $\begin{array}{c}\text { Max. } \\
\text { depth } \\
\text { (m) } \\
\end{array}$ & $\begin{array}{l}\text { Sampling } \\
\text { methods }\end{array}$ \\
\hline$\overline{1^{\mathrm{A}}}$ & Lagoa Boa Vista & Albuquerque (1990) & $\begin{array}{c}21^{\circ} 57^{\prime} \mathrm{S} \text { and } \\
47^{\circ} 53^{\prime} \mathrm{W}\end{array}$ & Mogi-Guaçu & 5.60 & 2.1 & $\begin{array}{c}\text { Sediment } \\
\text { (Ekman-Birge and } \\
\text { Hand Screen } \\
\text { Collector) } \\
\end{array}$ \\
\hline $1^{\mathrm{B}}$ & Lagoa Boa Vista & $\begin{array}{l}\text { Trivinho-Strixino \& } \\
\text { Strixino (1991a) }\end{array}$ & $\begin{array}{c}22^{\circ} 10^{\prime} \mathrm{S} \text { and } \\
47^{\circ} 54^{\prime} \mathrm{W} \\
\end{array}$ & Mogi-Guaçu & 5.60 & 2.1 & $\begin{array}{c}\text { Sediment } \\
\text { (Ekman-Birge) } \\
\end{array}$ \\
\hline $1^{\mathrm{C}}$ & Lagoa Boa Vista & $\begin{array}{l}\text { Trivinho-Strixino } \\
(1997)\end{array}$ & $\begin{array}{c}21^{\circ} 56^{\prime} 39.7^{\prime \prime} \mathrm{S} \text { and } \\
47^{\circ} 50^{\prime} 05.9^{\prime}, \mathrm{W} \\
\end{array}$ & Mogi-Guaçu & 5.60 & 2.1 & $\begin{array}{c}\text { Sediment } \\
\text { (Ekman-Birge) }\end{array}$ \\
\hline $1^{\mathrm{D}}$ & Lagoa Boa Vista & $\begin{array}{l}\text { Strixino \& } \\
\text { Trivinho-Strixino (1998) }\end{array}$ & $\begin{array}{c}21^{\circ} 57^{\prime} \mathrm{S} \text { and } \\
47^{\circ} 53^{\prime} \mathrm{W} \\
\end{array}$ & Mogi-Guaçu & 5.60 & 2.1 & $\begin{array}{c}\text { Sediment } \\
\text { (Ekman-Birge) }\end{array}$ \\
\hline $2^{\mathrm{A}}$ & $\begin{array}{c}\text { Lago Camargo } \\
\text { (Reservatório } \\
\text { Jurumirim) }\end{array}$ & $\begin{array}{l}\text { Stripari \& Henry } \\
(2002)\end{array}$ & $\begin{array}{c}23^{\circ} 27^{\prime} 30^{\prime \prime}- \\
23^{\circ} 30^{\prime} 00^{\prime \prime} \mathrm{S} \\
\text { and } \\
48^{\circ} 36 \text { ' } 50^{\prime \prime}- \\
48^{\circ} 38^{\prime} 29^{\prime}, \mathrm{W}\end{array}$ & Paranapanema & 22.45 & 3.9 & Litter bags \\
\hline $3^{\mathrm{A}}$ & Lagoa da Piaba & $\begin{array}{l}\text { Trivinho-Strixino et al. } \\
\text { (1998) }\end{array}$ & $\begin{array}{c}21^{\circ} 36^{\prime} 29.33^{\prime \prime S} \text { and } \\
47^{\circ} 49^{\prime} 48.9^{\prime \prime} \mathrm{W} \\
\end{array}$ & Mogi-Guaçu & 3.50 & - & $\begin{array}{c}\text { Macrophytes } \\
\text { (manual collection) } \\
\end{array}$ \\
\hline $3^{\mathrm{B}}$ & Lagoa da Piaba & $\begin{array}{l}\text { Strixino et al. } \\
\text { (1997) }\end{array}$ & $\begin{array}{c}21^{\circ} 36^{\prime} 29.3^{\prime \prime S} \text { and } \\
47^{\circ} 49^{\prime} 48.9^{\prime \prime} \mathrm{W}\end{array}$ & Mogi-Guaçu & 3.50 & - & $\begin{array}{c}\text { Sediment } \\
\text { (Ekman-Birge) }\end{array}$ \\
\hline $4^{\mathrm{A}}$ & Lagoa das Garças & $\begin{array}{l}\text { Santos \& Henry } \\
(2002)\end{array}$ & $\begin{array}{c}23^{\circ} 39^{\prime} \mathrm{S} \text { and } \\
46^{\circ} 37^{\prime} \mathrm{W}\end{array}$ & Alto Tietê & 8.86 & 4.6 & $\begin{array}{l}\text { Sediment } \\
\text { (Petersen) }\end{array}$ \\
\hline $5^{\mathrm{A}}$ & Lagoa do Diogo & $\begin{array}{l}\text { Alves \& Strixino (1997) } \\
\text { Alves (1998) }\end{array}$ & $\begin{array}{c}21^{\circ} 37^{\prime} 26.2^{\prime \prime} \mathrm{S} \text { and } \\
47^{\circ} 48^{\prime} 24.6^{\prime \prime} \mathrm{W} \\
\end{array}$ & Mogi-Guaçu & 69.00 & 3.0 & $\begin{array}{c}\text { Sediment } \\
\text { (Ekman-Birge) } \\
\end{array}$ \\
\hline $5^{\mathrm{B}}$ & Lagoa do Diogo & $\begin{array}{l}\text { Trivinho-Strixino et al. } \\
\text { (1998) }\end{array}$ & $\begin{array}{c}21^{\circ} 37^{\prime} 26.2^{\prime \prime} \mathrm{S} \text { and } \\
47^{\circ} 48^{\prime} 24.6^{\prime \prime} \mathrm{W}\end{array}$ & Mogi-Guaçu & 69.00 & 3.0 & $\begin{array}{c}\text { Macrophytes } \\
\text { (manual collection) } \\
\end{array}$ \\
\hline $5^{\mathrm{C}}$ & Lagoa do Diogo & $\begin{array}{l}\text { Strixino et al. } \\
(1997)\end{array}$ & $\begin{array}{c}21^{\circ} 37^{\prime} 26.2^{\prime \prime S} \text { and } \\
47^{\circ} 48^{\prime} 24.6^{\prime \prime} \mathrm{W} \\
\end{array}$ & Mogi-Guaçu & 69.00 & 3.0 & $\begin{array}{c}\text { Sediment } \\
(\text { Ekman-Birge) } \\
\end{array}$ \\
\hline $5^{\mathrm{D}}$ & Lagoa do Diogo & Alves \& Strixino (2000) & $\begin{array}{c}21^{\circ} 37^{\prime} 26.2^{\prime \prime} \mathrm{S} \text { and } \\
47^{\circ} 48^{\prime} 24.6^{\prime \prime} \mathrm{W}\end{array}$ & Mogi-Guaçu & 69.00 & 3.0 & $\begin{array}{c}\text { Sediment } \\
\text { (Ekman-Birge) }\end{array}$ \\
\hline $6^{\mathrm{A}}$ & Lagoa do Infernão & Correia (1996) & $\begin{array}{c}21^{\circ} 35^{\prime} \mathrm{S} \text { and } \\
47^{\circ} 51^{\prime} \mathrm{W} \\
\end{array}$ & Mogi-Guaçu & 3.18 & 4.0 & $\begin{array}{c}\text { Macrophytes } \\
\text { (manual collection) } \\
\end{array}$ \\
\hline $6^{\mathrm{B}}$ & Lagoa do Infernão & $\begin{array}{l}\text { Correia \& } \\
\text { Trivinho-Strixino (1998) }\end{array}$ & $\begin{array}{c}21^{\circ} 35^{\prime} \mathrm{S} \text { and } \\
47^{\circ} 51^{\prime} \mathrm{W} \\
\end{array}$ & Mogi-Guaçu & 3.18 & 4.0 & $\begin{array}{c}\text { Macrophytes } \\
\text { (manual collection) } \\
\end{array}$ \\
\hline $6^{\mathrm{C}}$ & Lagoa do Infernão & $\begin{array}{l}\text { Trivinho-Strixino et al. } \\
(1998)\end{array}$ & $\begin{array}{c}21^{\circ} 35^{\prime} \mathrm{S} \text { and } \\
47^{\circ} 51^{\prime} \mathrm{W} \\
\end{array}$ & Mogi-Guaçu & 3.18 & 4.0 & $\begin{array}{c}\text { Macrophytes } \\
\text { (manual collection) } \\
\end{array}$ \\
\hline $6^{\mathrm{D}}$ & Lagoa do Infernão & $\begin{array}{l}\text { Sonoda (1999) and Sonoda } \\
\text { \& Trivinho-Strixino (2000) }\end{array}$ & $\begin{array}{c}21^{\circ} 35^{\prime} \mathrm{S} \text { and } \\
47^{\circ} 51^{\prime} \mathrm{W} \\
\end{array}$ & Mogi-Guaçu & 3.18 & 4.0 & $\begin{array}{c}\text { Macrophytes } \\
\text { (manual collection) } \\
\end{array}$ \\
\hline $6^{\mathrm{E}}$ & Lagoa do Infernão & $\begin{array}{l}\text { Trivinho-Strixino \& } \\
\text { Strixino (2000a) }\end{array}$ & $\begin{array}{c}21^{\circ} 35^{\prime} \mathrm{S} \text { and } \\
47^{\circ} 51^{\prime} \mathrm{W} \\
\end{array}$ & Mogi-Guaçu & 3.18 & 4.0 & $\begin{array}{c}\text { Macrophytes } \\
\text { (manual collection) }\end{array}$ \\
\hline $6^{\mathrm{F}}$ & Lagoa do Infernão & $\begin{array}{l}\text { Trivinho-Strixino et al. } \\
\text { (2000) }\end{array}$ & $\begin{array}{c}21^{\circ} 35^{\prime} \mathrm{S} \text { and } \\
47^{\circ} 51^{\prime} \mathrm{W}\end{array}$ & Mogi-Guaçu & 3.18 & 4.0 & $\begin{array}{c}\text { Macrophytes } \\
\text { (manual collection) }\end{array}$ \\
\hline $77^{\mathrm{A}}$ & Lagoa do Óleo & $\begin{array}{l}\text { Strixino et al. } \\
(1997)\end{array}$ & $\begin{array}{c}21^{\circ} 35^{\prime} 26.9 \mathrm{~S}^{\prime \prime} \text { and } \\
47^{\circ} 50^{\prime} 12.0^{\prime \prime} \mathrm{W} \\
\end{array}$ & Mogi-Guaçu & 2.21 & - & $\begin{array}{c}\text { Macrophytes } \\
\text { (manual collection) } \\
\end{array}$ \\
\hline $7^{\mathrm{B}}$ & Lagoa do Óleo & $\begin{array}{l}\text { Trivinho-Strixino et al. } \\
\text { (1998) }\end{array}$ & $\begin{array}{c}21^{\circ} 35^{\prime} 26.9 \mathrm{~S}^{\prime \prime} \text { and } \\
47^{\circ} 50^{\prime} 12.0^{\prime} \text { 'W }\end{array}$ & Mogi-Guaçu & 2.21 & - & $\begin{array}{c}\text { Sediment } \\
(\text { Ekman-Birge) }\end{array}$ \\
\hline $8^{\mathrm{A}}$ & Lago dos Patos & Roque (1999) & $\begin{array}{c}23^{\circ} 27^{\prime} \mathrm{S} \text { and } \\
46^{\circ} 45^{\prime} \mathrm{W}\end{array}$ & Alto-Tietê & - & 1.0 & $\begin{array}{c}\text { Sediment } \\
\text { (Ekman-Birge) }\end{array}$ \\
\hline
\end{tabular}




\begin{tabular}{|c|c|c|c|c|c|c|c|}
\hline $9^{A}$ & Lagoa Dourada & $\begin{array}{l}\text { Melão \& Rocha } \\
\text { (1996) }\end{array}$ & $\begin{array}{c}17^{\circ} 55^{\prime} 2^{\prime \prime} \mathrm{S} \text { and } \\
22^{\circ} 11^{\prime} 33^{\prime \prime} \mathrm{W} \\
\end{array}$ & Tietê-Jacaré & 7.68 & 6.3 & $\begin{array}{c}\text { Freshwater } \\
\text { Sponges collection } \\
\end{array}$ \\
\hline $9^{\mathrm{B}}$ & Lagoa Dourada & Present study & $\begin{array}{c}17^{\circ} 55^{\prime} 2^{\prime \prime} \mathrm{S} \text { and } \\
22^{\circ} 11^{\prime} 33^{\prime \prime} \mathrm{W}\end{array}$ & Tietê-Jacaré & 7.68 & 6.3 & Exuviae Collection \\
\hline $10^{\mathrm{A}}$ & Lagoa Mayaca & $\begin{array}{l}\text { Borghoff-Gonçalves \& } \\
\text { Trivinho-Strixino (1997) }\end{array}$ & $\begin{array}{c}21^{\circ} 58^{\prime} 02.1^{\prime \prime} \mathrm{S} \text { and } \\
47^{\circ} 53^{\prime} 01.7^{\prime \prime} \mathrm{W}\end{array}$ & Tietê-Jacaré & 0.17 & 0.6 & Artificial Substrates \\
\hline $10^{\mathrm{B}}$ & Lagoa Mayaca & $\begin{array}{l}\text { Trivinho-Strixino \& } \\
\text { Strixino (2000a) }\end{array}$ & $\begin{array}{c}21^{\circ} 58^{\prime} 02.1^{\prime \prime} \mathrm{S} \text { and } \\
47^{\circ} 53^{\prime} 01.7^{\prime \prime} \mathrm{W} \\
\end{array}$ & Tietê-Jacaré & 0.17 & 0.6 & $\begin{array}{c}\text { Macrophytes } \\
\text { (manual collection) }\end{array}$ \\
\hline $10^{\mathrm{C}}$ & Lagoa Mayaca & $\begin{array}{l}\text { Trivinho-Strixino \& } \\
\text { Strixino }(2000 \mathrm{~b})\end{array}$ & $\begin{array}{c}21^{\circ} 58^{\prime} 02.1^{\prime \prime} \mathrm{S} \text { and } \\
47^{\circ} 53^{\prime} 01.7^{\prime \prime} \mathrm{W} \\
\end{array}$ & Tietê-Jacaré & 0.17 & 0.6 & $\begin{array}{c}\text { Macrophytes } \\
\text { (manual collection) } \\
\end{array}$ \\
\hline $10^{\mathrm{D}}$ & Lagoa Mayaca & $\begin{array}{l}\text { Trivinho-Strixino \& } \\
\text { Strixino (2003) }\end{array}$ & $\begin{array}{c}21^{\circ} 58^{\prime} 02.1^{\prime \prime S} \text { and } \\
47^{\circ} 53^{\prime} 01.7^{\prime \prime} \mathrm{W}\end{array}$ & Tietê-Jacaré & 0.17 & 0.6 & $\begin{array}{c}\text { Macrophytes } \\
\text { (manual collection) }\end{array}$ \\
\hline $11^{\mathrm{A}}$ & $\begin{array}{l}\text { Lago Camargo; Lago } \\
\text { Coqueiral; Lago } \\
\text { Cavalos (Reservatório } \\
\text { Jurumirim) }\end{array}$ & Afonso (2002) & $\begin{array}{c}23^{\circ} 27^{\prime} 30^{\prime \prime}- \\
23^{\circ} 30^{\prime} 00^{\prime \prime \prime S} \text { and } \\
48^{\circ} 36^{\prime} 50^{\prime \prime}- \\
48^{\circ} 38^{\prime} 29^{\prime \prime} \mathrm{W}\end{array}$ & Paranapaema & $\begin{array}{c}22.45 \\
64.13 \\
0.86\end{array}$ & $\begin{array}{l}3.9 \\
3.5 \\
2.4\end{array}$ & $\begin{array}{c}\text { Macrophytes } \\
\text { (manual collection) }\end{array}$ \\
\hline $12^{\mathrm{A}}$ & $\begin{array}{l}\text { Pisciculture tanks } \\
\text { (UFSCar- S. Carlos) }\end{array}$ & $\begin{array}{l}\text { Borghoff-Gonçalves \& } \\
\text { Trivinho-Strixino (1997) }\end{array}$ & $\begin{array}{c}21^{\circ} 59^{\prime} \mathrm{S} \text { and } \\
47^{\circ} 54^{\prime} \mathrm{W}\end{array}$ & Tietê-Jacaré & 0.10 & 1.0 & Artificial Substrates \\
\hline $13^{\mathrm{A}}$ & $\begin{array}{l}\text { Pisciculture tanks } \\
\text { (CEPTA- IBAMA- } \\
\text { Pirassununga) }\end{array}$ & Campos (1999) & $\begin{array}{c}21^{\circ} 55^{\prime} 44.5^{\prime \prime S} \text { and } \\
47^{\circ} 22^{\prime} 23.7^{\prime \prime} \mathrm{W}\end{array}$ & Mogi-Guaçu & 0.10 & 1.0 & $\begin{array}{c}\text { Sediment } \\
\text { (Ekman-Birge) }\end{array}$ \\
\hline $14^{\mathrm{A}}$ & Represa Nova & Campos (1999) & $\begin{array}{c}21^{\circ} 55^{\prime} 44.5^{\prime \prime} \mathrm{S} \text { and } \\
47^{\circ} 22^{\prime} 23.7^{\prime \prime} \mathrm{W}\end{array}$ & Mogi-Guaçu & - & 5.0 & $\begin{array}{c}\text { Sediment } \\
\text { (Ekman-Birge) }\end{array}$ \\
\hline $15^{\mathrm{A}}$ & $\begin{array}{c}\text { Reservatório Álvaro } \\
\text { de Souza Lima (Bariri) }\end{array}$ & $\begin{array}{l}\text { Rodrigues } \\
\text { (Personal communication) }\end{array}$ & $\begin{array}{l}22^{\circ} 9^{\prime} 49^{\prime \prime S} \text { and } \\
48^{\circ} 44^{\prime} 20.8^{\prime \prime} \mathrm{W}\end{array}$ & Médio Tietê & 6300.00 & 32.5 & $\begin{array}{c}\text { Sediment } \\
\text { (Ekman-Birge) }\end{array}$ \\
\hline $16^{\mathrm{A}}$ & Reservatório Billings & Kuhlmann et al. (1997) & $\begin{array}{c}23^{\circ} 42^{\prime} \mathrm{S} \text { and } \\
46^{\circ} 22^{\prime} \mathrm{W}\end{array}$ & Alto Tietê & 12000.00 & 15.0 & $\begin{array}{c}\text { Sediment } \\
\text { (Ekman-Birge and } \\
\text { Petersen) }\end{array}$ \\
\hline $17^{\mathrm{A}}$ & $\begin{array}{c}\text { Reservatório } \\
\text { da Colônia }\end{array}$ & $\begin{array}{l}\text { Strixino \& } \\
\text { Trivinho-Strixino (1998) }\end{array}$ & $\begin{array}{c}21^{\circ} 58^{\prime} \mathrm{S} \text { and } \\
47^{\circ} 53^{\prime} \mathrm{W} \\
\end{array}$ & Mogi-Guaçu & 0.30 & 3.5 & $\begin{array}{c}\text { Sediment } \\
\text { (Ekman-Birge) }\end{array}$ \\
\hline $17^{\mathrm{B}}$ & $\begin{array}{l}\text { Reservatório } \\
\text { da Colônia }\end{array}$ & $\begin{array}{l}\text { Trivinho-Strixino \& } \\
\text { Strixino (2003) }\end{array}$ & $\begin{array}{c}21^{\circ} 58^{\prime} \mathrm{S} \text { and } \\
47^{\circ} 53^{\prime} \mathrm{W}\end{array}$ & Mogi-Guaçu & 0.30 & 3.5 & $\begin{array}{c}\text { Sediment } \\
\text { (Ekman-Birge and } \\
\text { Hand Screen } \\
\text { Collector) } \\
\end{array}$ \\
\hline $18^{\mathrm{A}}$ & $\begin{array}{c}\text { Reservatório } \\
\text { da Mata }\end{array}$ & $\begin{array}{l}\text { Strixino \& } \\
\text { Trivinho-Strixino (1998) }\end{array}$ & $\begin{array}{c}21^{\circ} 58^{\prime} \mathrm{S} \text { and } \\
47^{\circ} 53^{\prime} \mathrm{W} \\
\end{array}$ & Mogi-Guaçu & 0.20 & 2.5 & $\begin{array}{c}\text { Sediment } \\
\text { (Ekman-Birge) } \\
\end{array}$ \\
\hline $18^{\mathrm{B}}$ & $\begin{array}{l}\text { Reservatório } \\
\text { da Mata }\end{array}$ & $\begin{array}{l}\text { Trivinho-Strixino \& } \\
\text { Strixino (1991b) and } \\
\text { Trivinho-Strixino \& } \\
\text { Sanseverino (2003) } \\
\end{array}$ & $\begin{array}{l}21^{\circ} 58^{\prime} \mathrm{S} \text { and } \\
47053^{\prime} \mathrm{W}\end{array}$ & Mogi-Guaçu & 0.20 & 2.5 & $\begin{array}{c}\text { Sediment } \\
\text { (Ekman-Birge and } \\
\text { Hand Screen } \\
\text { Collector) } \\
\end{array}$ \\
\hline $18^{\mathrm{C}}$ & $\begin{array}{l}\text { Reservatório } \\
\text { da Mata }\end{array}$ & $\begin{array}{l}\text { Trivinho-Strixino \& } \\
\text { Strixino (2003) }\end{array}$ & $\begin{array}{c}21^{\circ} 58^{\prime} \mathrm{S} \text { and } \\
47^{\circ} 53^{\prime} \mathrm{W}\end{array}$ & Mogi-Guaçu & 0.20 & 2.5 & $\begin{array}{c}\text { Sediment } \\
\text { (Ekman-Birge and } \\
\text { Hand Screen } \\
\text { Collector) }\end{array}$ \\
\hline $19^{\mathrm{A}}$ & $\begin{array}{c}\text { Reservatório Monte } \\
\text { Alegre }\end{array}$ & $\begin{array}{l}\text { H. Mendes } \\
\text { (Personal communication) }\end{array}$ & $\begin{array}{c}21^{\circ} 11^{\prime} \mathrm{S} \text { and } \\
47^{\circ} 51^{\prime} \mathrm{W}\end{array}$ & Rio Pardo & 7.00 & 5.0 & $\begin{array}{c}\text { Sediment } \\
\text { (Ekman-Birge) and } \\
\text { adult collections } \\
\end{array}$ \\
\hline $20^{\mathrm{A}}$ & $\begin{array}{l}\text { Reservatório } \\
\text { de Americana }\end{array}$ & Shimizu (1978) & $\begin{array}{c}22^{\circ} 44^{\prime} \mathrm{S} \text { and } \\
47^{\circ} 20^{\prime} \mathrm{W} \\
\end{array}$ & Piracicaba & 1350.00 & 19.0 & $\begin{array}{c}\text { Sediment } \\
\text { (Ekman-Birge) }\end{array}$ \\
\hline $20^{\mathrm{B}}$ & $\begin{array}{l}\text { Reservatório } \\
\text { de Americana }\end{array}$ & Shimizu (1981) & $\begin{array}{c}22^{\circ} 44^{\prime} \mathrm{S} \text { and } \\
47^{\circ} 20^{\prime} \mathrm{W} \\
\end{array}$ & Piracicaba & 1350.00 & 19.0 & $\begin{array}{c}\text { Sediment } \\
\text { (Ekman-Birge) }\end{array}$ \\
\hline $20^{\mathrm{C}}$ & Reservatório & Pamplin (1999) & $22^{\circ} 44^{\prime} \mathrm{S}$ and & Piracicaba & 1350.00 & 19.0 & Sediment \\
\hline
\end{tabular}




\begin{tabular}{|c|c|c|c|c|c|c|c|}
\hline & de Americana & & $47^{\circ} 20^{\prime} \mathrm{W}$ & & & & (Ekman-Birge) \\
\hline $20^{\mathrm{D}}$ & $\begin{array}{l}\text { Reservatório } \\
\text { de Americana }\end{array}$ & Dornfeld (2002) & $\begin{array}{c}22^{\circ} 44^{\prime} \mathrm{S} \text { and } \\
47^{\circ} 20^{\prime} \mathrm{W}\end{array}$ & Piracicaba & 1350.00 & 19.0 & $\begin{array}{c}\text { Sediment } \\
\text { (Ekman-Birge) }\end{array}$ \\
\hline $21^{\mathrm{A}}$ & $\begin{array}{c}\text { Reservatório } \\
\text { de Barra Bonita }\end{array}$ & Soriano (1997) & $\begin{array}{c}22^{\circ} 29^{\prime} \mathrm{S}-22^{\circ} 44^{\prime} \mathrm{S} \\
\text { and } \\
48^{\circ} 34^{\prime}-48^{\circ} 10^{\prime} \mathrm{W}\end{array}$ & Médio Tietê & 31000.00 & 27.5 & $\begin{array}{c}\text { Sediment } \\
\text { (Ekman-Birge and } \\
\text { Hand Screen } \\
\text { Collector) }\end{array}$ \\
\hline $21^{\mathrm{B}}$ & $\begin{array}{c}\text { Reservatório } \\
\text { de Barra Bonita } \\
\end{array}$ & $\begin{array}{l}\text { Rodrigues } \\
\text { (Personal communication) }\end{array}$ & $\begin{array}{c}20^{\circ} 31^{\prime} \mathrm{S} \text { and } \\
48^{\circ} 32^{\prime} \mathrm{W} \\
\end{array}$ & Médio Tietê & 31000.00 & 32.5 & $\begin{array}{c}\text { Sediment } \\
\text { (Ekman-Birge) } \\
\end{array}$ \\
\hline $22^{\mathrm{A}}$ & $\begin{array}{c}\text { Reservatório } \\
\text { Quinta da Felicidade }\end{array}$ & $\begin{array}{l}\text { Trivinho-Strixino \& } \\
\text { Strixino (1991b) and } \\
\text { Trivinho-Strixino \& } \\
\text { Sanseverino (2003) }\end{array}$ & $\begin{array}{c}21^{\circ} 57^{\prime} \mathrm{S} \text { and } \\
47^{\circ} 54^{\prime} \mathrm{W}\end{array}$ & Mogi-Guaçu & 0.20 & 2.5 & $\begin{array}{c}\text { Sediment } \\
\text { (Ekman-Birge) }\end{array}$ \\
\hline $22^{\mathrm{B}}$ & $\begin{array}{c}\text { Reservatório } \\
\text { Quinta da Felicidade }\end{array}$ & $\begin{array}{l}\text { Strixino \& } \\
\text { Trivinho-Strixino (1998) }\end{array}$ & $\begin{array}{c}21^{\circ} 57^{\prime} \mathrm{S} \text { and } \\
47^{\circ} 54^{\prime} \mathrm{W} \\
\end{array}$ & Mogi-Guaçu & 0.20 & 2.5 & $\begin{array}{c}\text { Sediment } \\
\text { (Ekman-Birge) } \\
\end{array}$ \\
\hline $23^{A}$ & $\begin{array}{c}\text { Reservatório } \\
\text { de Ibitinga }\end{array}$ & $\begin{array}{l}\text { Rodrigues } \\
\text { (Personal communication) }\end{array}$ & $\begin{array}{c}21^{\circ} 45^{\prime} \mathrm{S} \text { and } \\
48^{\circ} 59^{\prime} \mathrm{W}\end{array}$ & Médio Tietê & 5600.00 & 31.7 & $\begin{array}{c}\text { Sediment } \\
\text { (Ekman-Birge) }\end{array}$ \\
\hline $24^{\mathrm{A}}$ & $\begin{array}{c}\text { Reservatório } \\
\text { de Jupiá }\end{array}$ & Roque (present study) & $\begin{array}{c}24^{\circ} 48^{\prime} \mathrm{S} \text { and } \\
51^{\circ} 38^{\prime} \mathrm{W}\end{array}$ & Paraná & 54400.00 & 30.0 & $\begin{array}{c}\text { Sediment } \\
\text { (Ekman-Birge) }\end{array}$ \\
\hline $25^{\mathrm{A}}$ & $\begin{array}{c}\text { Reservatório } \\
\text { de Ponte Nova }\end{array}$ & Pamplin (2002) & $\begin{array}{c}23^{\circ} 30^{\prime} \mathrm{S} \text { and } \\
46^{\circ} 00^{\prime} \mathrm{S}\end{array}$ & Alto Tietê & 2568.00 & 17.0 & $\begin{array}{c}\text { Sediment } \\
\text { (Van-Veen) }\end{array}$ \\
\hline $26^{\mathrm{A}}$ & $\begin{array}{l}\text { Reservatório } \\
\text { de Paraibuna }\end{array}$ & Kuhlmann (1993) & $\begin{array}{l}22^{\circ} 45^{\prime}-23^{\circ} 35^{\prime} \mathrm{S} \\
\text { and } \\
44^{\circ} 45^{\prime}-45^{\circ} 40^{\prime} \mathrm{W}\end{array}$ & $\begin{array}{l}\text { Paraíba } \\
\text { do Sul }\end{array}$ & 18800.00 & 90.0 & $\begin{array}{c}\text { Sediment } \\
\text { (Ekman-Birge) }\end{array}$ \\
\hline $26^{\mathrm{B}}$ & $\begin{array}{l}\text { Reservatório } \\
\text { de Paraibuna }\end{array}$ & Anaya et al. (1997) & $\begin{array}{c}22^{\circ} 45^{\prime}-23^{\circ} 35^{\prime} \mathrm{S} \\
\text { and } \\
44^{\circ} 45^{\prime}-45^{\circ} 40^{\prime} \mathrm{W}\end{array}$ & $\begin{array}{l}\text { Paraíba } \\
\text { do Sul }\end{array}$ & 18800.00 & 90.0 & $\begin{array}{l}\text { Sediment } \\
\text { (Petersen) }\end{array}$ \\
\hline $27^{A}$ & $\begin{array}{l}\text { Reservatório } \\
\text { de Promissão }\end{array}$ & $\begin{array}{l}\text { Rodrigues } \\
\text { (Personal communication) }\end{array}$ & $\begin{array}{c}21^{\circ} 18^{\prime} \mathrm{S} \text { and } \\
49^{\circ} 47^{\prime} \mathrm{W}\end{array}$ & Médio Tietê & 53000.00 & - & $\begin{array}{c}\text { Sediment } \\
\text { (Ekman-Birge) }\end{array}$ \\
\hline $28^{\mathrm{A}}$ & $\begin{array}{l}\text { Reservatório } \\
\text { do Beija-Flor }\end{array}$ & Rodrigues (1997) & $\begin{array}{c}21^{\circ} 36^{\prime} 01.5^{\prime} \text { 'S and } \\
47^{\circ} 47^{\prime} 03.0^{\prime \prime} \mathrm{W}\end{array}$ & Mogi-Guaçu & 17.54 & 2.4 & $\begin{array}{c}\text { Sediment } \\
\text { (Ekman-Birge) }\end{array}$ \\
\hline $28^{\mathrm{B}}$ & $\begin{array}{l}\text { Reservatório } \\
\text { do Beija-Flor }\end{array}$ & $\begin{array}{l}\text { Trivinho-Strixino et al. } \\
\text { (1998) }\end{array}$ & $\begin{array}{c}21^{\circ} 36^{\prime} 01.5^{\prime \prime} \mathrm{S} \text { and } \\
47^{\circ} 47^{\prime} 03.0 \text { " W }\end{array}$ & Mogi-Guaçu & 17.54 & 2.4 & $\begin{array}{c}\text { Macrophytes } \\
\text { (manual collection) }\end{array}$ \\
\hline $28^{\mathrm{C}}$ & $\begin{array}{l}\text { Reservatório } \\
\text { do Beija-Flor }\end{array}$ & Sonoda (1999) & $\begin{array}{c}21^{\circ} 36^{\prime} 01.5^{\prime \prime} \mathrm{S} \text { and } \\
47^{\circ} 47^{\prime} 03.0^{\prime \prime} \mathrm{W}\end{array}$ & Mogi-Guaçu & 17.54 & 2.4 & $\begin{array}{c}\text { Macrophytes } \\
\text { (manual collection) }\end{array}$ \\
\hline $28^{\mathrm{D}}$ & $\begin{array}{l}\text { Reservatório } \\
\text { do Beija-Flor }\end{array}$ & $\begin{array}{l}\text { Trivinho-Strixino \& } \\
\text { Strixino (2000a) }\end{array}$ & $\begin{array}{c}21^{\circ} 36^{\prime} 01.5^{\prime \prime} \mathrm{S} \text { and } \\
47^{\circ} 47^{\prime} 03.0^{\prime \prime} \mathrm{W}\end{array}$ & Mogi-Guaçu & 17.54 & 2.4 & $\begin{array}{c}\text { Decayed woods } \\
\text { (manual collection) }\end{array}$ \\
\hline $28^{\mathrm{E}}$ & $\begin{array}{l}\text { Reservatório } \\
\text { do Beija-Flor }\end{array}$ & Strixino et al. (1997) & $\begin{array}{c}21^{\circ} 36^{\prime} 01.5^{\prime} \text { 'S and } \\
47^{\circ} 47^{\prime} 03.0^{\prime \prime} \mathrm{W} \\
\end{array}$ & Mogi-Guaçu & 17.54 & 2.4 & $\begin{array}{c}\text { Sediment } \\
\text { (Ekman-Birge) } \\
\end{array}$ \\
\hline $29^{\mathrm{A}}$ & $\begin{array}{c}\text { Reservatório } \\
\text { do Fazzari }\end{array}$ & Albuquerque (1990) & $\begin{array}{c}21^{\circ} 59^{\prime} \mathrm{S} \text { and } \\
47^{\circ} 54^{\prime} \mathrm{W}\end{array}$ & Tietê-Jacaré & 1.30 & 4.0 & $\begin{array}{c}\text { Sediment } \\
\text { (Ekman-Birge and } \\
\text { Hand Screen } \\
\text { Collector) } \\
\end{array}$ \\
\hline $29^{\mathrm{B}}$ & $\begin{array}{c}\text { Reservatório } \\
\text { do Fazzari }\end{array}$ & $\begin{array}{l}\text { Trivinho-Strixino \& } \\
\text { Strixino (1991b) and } \\
\text { Trivinho-Strixino \& } \\
\text { Sanseverino (2003) }\end{array}$ & $\begin{array}{c}21^{\circ} 59^{\prime} \mathrm{S} \text { and } \\
47^{\circ} 54^{\prime} \mathrm{W}\end{array}$ & Tietê-Jacaré & 1.30 & 4.0 & $\begin{array}{c}\text { Sediment } \\
\text { (Ekman-Birge and } \\
\text { Hand Screen } \\
\text { Collector) }\end{array}$ \\
\hline $29^{\mathrm{C}}$ & $\begin{array}{c}\text { Reservatório } \\
\text { do Fazzari }\end{array}$ & $\begin{array}{l}\text { Borghoff-Gonçalves \& } \\
\text { Trivinho-Strixino (1997) }\end{array}$ & $\begin{array}{c}21^{\circ} 59^{\prime} \mathrm{S} \text { and } \\
47^{\circ} 54^{\prime} \mathrm{W} \\
\end{array}$ & Tietê-Jacaré & 1.30 & 4.0 & Artificial Substrates \\
\hline $29^{\mathrm{D}}$ & $\begin{array}{c}\text { Reservatório } \\
\text { do Fazzari }\end{array}$ & $\begin{array}{l}\text { Strixino \& } \\
\text { Trivinho-Strixino (1998) }\end{array}$ & $\begin{array}{c}21^{\circ} 59^{\prime} \mathrm{S} \text { and } \\
47^{\circ} 54^{\prime} \mathrm{W}\end{array}$ & Tietê-Jacaré & 1.30 & 4.0 & $\begin{array}{c}\text { Sediment } \\
\text { (Ekman-Birge) }\end{array}$ \\
\hline $29^{\mathrm{E}}$ & $\begin{array}{c}\text { Reservatório } \\
\text { do Fazzari }\end{array}$ & $\begin{array}{l}\text { Trivinho-Strixino \& } \\
\text { Strixino (1998a) }\end{array}$ & $\begin{array}{c}21^{\circ} 59^{\prime} \mathrm{S} \text { and } \\
47^{\circ} 54^{\prime} \mathrm{W}\end{array}$ & Tietê-Jacaré & 1.30 & 4.0 & $\begin{array}{c}\text { Decayed woods } \\
\text { (manual collection) } \\
\text { Sediment } \\
\text { (Ekman-Birge) }\end{array}$ \\
\hline
\end{tabular}

http://www.biotaneotropica.org.br 


\begin{tabular}{|c|c|c|c|c|c|c|c|}
\hline $29^{\mathrm{F}}$ & $\begin{array}{c}\text { Reservatório } \\
\text { do Fazzari }\end{array}$ & $\begin{array}{l}\text { Trivinho-Strixino \& } \\
\text { Strixino (2000a) }\end{array}$ & $\begin{array}{c}21^{\circ} 59^{\prime} \mathrm{S} \text { and } \\
47^{\circ} 54^{\prime} \mathrm{W} \\
\end{array}$ & Tietê-Jacaré & 1.30 & 4.0 & $\begin{array}{c}\text { Decayed woods } \\
\text { (manual collection) }\end{array}$ \\
\hline $29^{\mathrm{G}}$ & $\begin{array}{c}\text { Reservatório } \\
\text { do Fazzari }\end{array}$ & $\begin{array}{l}\text { Trivinho-Strixino \& } \\
\text { Strixino (2003) }\end{array}$ & $\begin{array}{c}21^{\circ} 59^{\prime} \mathrm{S} \text { and } \\
47^{\circ} 54^{\prime} \mathrm{W}\end{array}$ & Tietê-Jacaré & 1.30 & 4.0 & $\begin{array}{c}\text { Sediment } \\
\text { (Ekman-Birge and } \\
\text { Hand Screen } \\
\text { Collector) }\end{array}$ \\
\hline $30^{\mathrm{A}}$ & $\begin{array}{c}\text { Reservatório } \\
\text { do Jaraguá }\end{array}$ & Roque (1999) & $\begin{array}{c}23^{\circ} 27^{\prime} 78^{\prime \prime} \mathrm{S} \text { and } \\
46^{\circ} 45^{\prime} 60^{\prime \prime} \mathrm{W} ?\end{array}$ & Alto-Tietê & - & 2.5 & $\begin{array}{c}\text { Sediment } \\
\text { (Ekman-Birge) } \\
\end{array}$ \\
\hline $31^{\mathrm{A}}$ & $\begin{array}{l}\text { Reservatório } \\
\text { do Lobo }\end{array}$ & Strixino (1973) & $\begin{array}{c}22^{\circ} 10^{\prime} \mathrm{S} \text { and } \\
47^{\circ} 54^{\prime} \mathrm{W}\end{array}$ & Tietê-Jacaré & 680.00 & 11.0 & $\begin{array}{c}\text { Sediment } \\
\text { (Ekman-Birge) }\end{array}$ \\
\hline $31^{\mathrm{B}}$ & $\begin{array}{c}\text { Reservatório } \\
\text { do Lobo }\end{array}$ & $\begin{array}{l}\text { Trivinho-Strixino \& } \\
\text { Strixino (1991a) } \\
\end{array}$ & $\begin{array}{c}22^{\circ} 10^{\prime} \mathrm{S} \text { and } \\
47^{\circ} 54^{\prime} \mathrm{W} \\
\end{array}$ & Tietê-Jacaré & 680.00 & 11.0 & $\begin{array}{c}\text { Macrophytes } \\
\text { (manual collection) }\end{array}$ \\
\hline $31^{\mathrm{C}}$ & $\begin{array}{l}\text { Reservatório } \\
\text { do Lobo }\end{array}$ & $\begin{array}{l}\text { Trivinho-Strixino \& } \\
\text { Strixino (1991b) and } \\
\text { Trivinho-Strixino \& } \\
\text { Sanseverino (2003) } \\
\end{array}$ & $\begin{array}{c}22^{\circ} 10^{\prime} \mathrm{S} \text { and } \\
47^{\circ} 54^{\prime} \mathrm{W}\end{array}$ & Tietê-Jacaré & 680.00 & 11.0 & $\begin{array}{c}\text { Sediment } \\
\text { (Ekman-Birge) }\end{array}$ \\
\hline $31^{\mathrm{D}}$ & $\begin{array}{c}\text { Reservatório } \\
\text { do Lobo }\end{array}$ & $\begin{array}{l}\text { Trivinho-Strixino \& } \\
\text { Strixino (1993) }\end{array}$ & $\begin{array}{c}22^{\circ} 10^{\prime} \mathrm{S} \text { and } \\
47^{\circ} 54^{\prime} \mathrm{W}\end{array}$ & Tietê-Jacaré & 680.00 & 11.0 & $\begin{array}{c}\text { Macrophytes } \\
\text { (manual collection) }\end{array}$ \\
\hline $31^{\mathrm{E}}$ & $\begin{array}{c}\text { Reservatório } \\
\text { do Lobo }\end{array}$ & Camargo (1984) & $\begin{array}{c}22^{\circ} 10^{\prime} \mathrm{S} \text { and } \\
47^{\circ} 54^{\prime} \mathrm{W} \\
\end{array}$ & Tietê-Jacaré & 680.00 & 11.0 & $\begin{array}{c}\text { Macrophytes } \\
\text { (manual collection) }\end{array}$ \\
\hline $31^{\mathrm{F}}$ & $\begin{array}{c}\text { Reservatório } \\
\text { do Lobo }\end{array}$ & $\begin{array}{l}\text { Strixino \& } \\
\text { Trivinho-Strixino (1998) }\end{array}$ & $\begin{array}{c}22^{\circ} 10^{\prime} \mathrm{S} \text { and } \\
47^{\circ} 54^{\prime} \mathrm{W} \\
\end{array}$ & Tietê-Jacaré & 680.00 & 11.0 & $\begin{array}{c}\text { Sediment } \\
\text { (Ekman-Birge) }\end{array}$ \\
\hline $31^{\mathrm{G}}$ & $\begin{array}{c}\text { Reservatório } \\
\text { do Lobo }\end{array}$ & $\begin{array}{l}\text { Trivinho-Strixino \& } \\
\text { Strixino (2000a) }\end{array}$ & $\begin{array}{c}22^{\circ} 10^{\prime} \mathrm{S} \text { and } \\
47^{\circ} 54^{\prime} \mathrm{W} \\
\end{array}$ & Tietê-Jacaré & 680.00 & 11.0 & $\begin{array}{c}\text { Macrophytes } \\
\text { (manual collection) }\end{array}$ \\
\hline $32^{\mathrm{A}}$ & $\begin{array}{c}\text { Reservatório } \\
\text { do Monjolinho }\end{array}$ & Strixino \& Strixino (1982) & $\begin{array}{c}22^{\circ} 00^{\prime} \mathrm{S} \text { and } \\
47^{\circ} 54^{\prime} \mathrm{W} \\
\end{array}$ & Tietê-Jacaré & 4.70 & 3.0 & $\begin{array}{c}\text { Sediment } \\
(\text { Ekman-Birge })\end{array}$ \\
\hline $32^{\mathrm{B}}$ & $\begin{array}{c}\text { Reservatório } \\
\text { do Monjolinho }\end{array}$ & Strixino \& Strixino (1984) & $\begin{array}{c}22^{\circ} 00^{\prime} \mathrm{S} \text { and } \\
47^{\circ} 54^{\prime} \mathrm{W} \\
\end{array}$ & Tietê-Jacaré & 4.70 & 3.0 & $\begin{array}{c}\text { Macrophytes } \\
\text { (manual collection) }\end{array}$ \\
\hline $32^{\mathrm{C}}$ & $\begin{array}{c}\text { Reservatório } \\
\text { do Monjolinho }\end{array}$ & $\begin{array}{l}\text { Strixino \& } \\
\text { Trivinho-Strixino (1998) }\end{array}$ & $\begin{array}{c}22^{\circ} 00^{\prime} \mathrm{S} \text { and } \\
47^{\circ} 54^{\prime} \mathrm{W} \\
\end{array}$ & Tietê-Jacaré & 4.70 & 4.0 & $\begin{array}{c}\text { Sediment } \\
(\text { Ekman-Birge }) \\
\end{array}$ \\
\hline $32^{\mathrm{D}}$ & $\begin{array}{c}\text { Reservatório } \\
\text { do Monjolinho }\end{array}$ & $\begin{array}{l}\text { Trivinho-Strixino \& } \\
\text { Strixino }(1998 b)\end{array}$ & $\begin{array}{c}22^{\circ} 00^{\prime} \mathrm{S} \text { and } \\
47^{\circ} 54^{\prime} \mathrm{W} \\
\end{array}$ & Tietê-Jacaré & 4.70 & 3.0 & $\begin{array}{c}\text { Sediment } \\
\text { (Ekman-Birge) } \\
\end{array}$ \\
\hline $32^{\mathrm{E}}$ & $\begin{array}{c}\text { Reservatório } \\
\text { do Monjolinho }\end{array}$ & Correia (1999) & $\begin{array}{l}22^{\circ} 00^{\prime} \mathrm{S} \text { and } \\
47^{\circ} 54^{\prime} \mathrm{W}\end{array}$ & Tietê-Jacaré & 4.70 & 3.0 & $\begin{array}{c}\text { Sediment } \\
\text { (Ekman-Birge) } \\
\text { Artificial Substrates; } \\
\text { Macrophytes } \\
\text { (manual collection) }\end{array}$ \\
\hline $32^{\mathrm{F}}$ & $\begin{array}{c}\text { Reservatório } \\
\text { do Monjolinho } \\
\end{array}$ & Léo (1999) & $\begin{array}{c}22^{\circ} 00^{\prime} \mathrm{S} \text { and } \\
47^{\circ} 54^{\prime} \mathrm{W} \\
\end{array}$ & Tietê-Jacaré & 4.70 & 3.0 & $\begin{array}{c}\text { Sediment } \\
(\text { Ekman-Birge }) \\
\end{array}$ \\
\hline $32^{\mathrm{G}}$ & $\begin{array}{l}\text { Reservatório } \\
\text { do Monjolinho }\end{array}$ & Barros (2002) & $\begin{array}{c}22^{\circ} 00^{\prime} \mathrm{S} \text { and } \\
47^{\circ} 54^{\prime} \mathrm{W}\end{array}$ & Tietê-Jacaré & 4.70 & 3.0 & $\begin{array}{l}\text { Drift and } \\
\text { manual nets }\end{array}$ \\
\hline $32^{\mathrm{H}}$ & $\begin{array}{c}\text { Reservatório } \\
\text { do Monjolinho }\end{array}$ & $\begin{array}{l}\text { Roque \& Trivinho- } \\
\text { Strixino (submitted) }\end{array}$ & $\begin{array}{c}22^{\circ} 00^{\prime} \mathrm{S} \text { and } \\
47^{\circ} 54^{\prime} \mathrm{W} \\
\end{array}$ & Tietê-Jacaré & 4.70 & 3.0 & $\begin{array}{c}\text { Adults colletion } \\
\text { (hand net) }\end{array}$ \\
\hline $32^{I}$ & $\begin{array}{c}\text { Reservatório } \\
\text { do Monjolinho }\end{array}$ & $\begin{array}{l}\text { Correia \& Trivinho- } \\
\text { Strixino (submitted a) }\end{array}$ & $\begin{array}{c}22^{\circ} 00^{\prime} \mathrm{S} \text { and } \\
47^{\circ} 54^{\prime} \mathrm{W} \\
\end{array}$ & Tietê-Jacaré & 4.70 & 3.0 & Artificial Substrates \\
\hline $32^{J}$ & $\begin{array}{c}\text { Reservatório } \\
\text { do Monjolinho }\end{array}$ & $\begin{array}{l}\text { Correia \& Trivinho- } \\
\text { Strixino (submitted b) }\end{array}$ & $\begin{array}{c}22^{\circ} 00^{\prime} \mathrm{S} \text { and } \\
47^{\circ} 54^{\prime} \mathrm{W}\end{array}$ & Tietê-Jacaré & 4.70 & 3.0 & Artificial Substrates \\
\hline $33^{\mathrm{A}}$ & $\begin{array}{c}\text { Reservatório } \\
\text { do Parque Ecológico } \\
\text { A. T. Vieira (S. Carlos) } \\
\end{array}$ & Dornfeld (1999) & - & Tietê-Jacaré & - & - & $\begin{array}{c}\text { Macrophytes } \\
\text { (manual collection) }\end{array}$ \\
\hline $34^{\mathrm{A}}$ & $\begin{array}{l}\text { Reservatório } \\
\text { do Pasto }\end{array}$ & $\begin{array}{l}\text { Strixino \& } \\
\text { Trivinho-Strixino (1998) }\end{array}$ & $\begin{array}{l}21^{\circ} 58^{\prime} \mathrm{S} \text { and } \\
47^{\circ} 53^{\prime} \mathrm{W}\end{array}$ & Mogi-Guaçu & 0.70 & 3.0 & $\begin{array}{c}\text { Sediment } \\
\text { (Ekman-Birge) }\end{array}$ \\
\hline
\end{tabular}




\begin{tabular}{|c|c|c|c|c|c|c|c|}
\hline $35^{\mathrm{A}}$ & $\begin{array}{c}\text { Reservatório do } \\
\text { Ribeirão das Anhumas }\end{array}$ & $\begin{array}{l}\text { Corbi (2001) and Corbi } \\
\& \text { Trivinho-Strixino (2002) }\end{array}$ & $\begin{array}{c}21^{\circ} 42^{\prime} \mathrm{S} \text { and } \\
48^{\circ} 00^{\prime \prime}\end{array}$ & Mogi-Guaçu & 82.00 & 7.6 & $\begin{array}{c}\text { Sediment } \\
\text { (Ekman-Birge) }\end{array}$ \\
\hline $36^{\mathrm{A}}$ & $\begin{array}{l}\text { Reservatório } \\
\text { Jacaré-Pepira }\end{array}$ & $\begin{array}{l}\text { Strixino \& } \\
\text { Trivinho-Strixino (1998) }\end{array}$ & $\begin{array}{c}22^{\circ} 26^{\prime} \mathrm{S} \text { and } \\
48^{\circ} 10^{\prime} \mathrm{W}\end{array}$ & Tietê-Jacaré & 129.00 & 11.5 & $\begin{array}{c}\text { Sediment } \\
\text { (Ekman-Birge) }\end{array}$ \\
\hline $37^{\mathrm{A}}$ & $\begin{array}{l}\text { Reservatório } \\
\text { Jurumirim }\end{array}$ & Santos \& Henry (2001) & $\begin{array}{c}23^{\circ} 12^{\prime} \mathrm{S} \text { and } \\
49^{\circ} 13^{\prime} \mathrm{W}\end{array}$ & $\begin{array}{c}\text { Alto } \\
\text { Paranapanema }\end{array}$ & 48480.00 & 40.0 & $\begin{array}{l}\text { Sedimento } \\
\text { (Pettersen) }\end{array}$ \\
\hline $38^{\mathrm{A}}$ & $\begin{array}{l}\text { Reservatório } \\
\text { Mogi-Guaçu }\end{array}$ & Brandimarte (1997) & $\begin{array}{c}22^{\circ} 19^{\prime}-22^{\circ} 24^{\prime} \mathrm{S} \text { and } \\
46^{\circ} 54^{\prime}-46^{\circ} 48^{\prime} \mathrm{W}\end{array}$ & Mogi-Guaçu & 573.00 & 13.5 & $\begin{array}{l}\text { Sediment } \\
\text { (Pettersen) }\end{array}$ \\
\hline $39^{\mathrm{A}}$ & Lagoa do Quilômetro & Strixino et al. (1997) & $\begin{array}{c}21^{\circ} 36^{\prime} 29.3^{\prime \prime} \mathrm{S} \text { and } \\
47^{\circ} 49^{\prime} 48.9^{\prime \prime} \mathrm{W}\end{array}$ & Mogi-Guaçu & 3.20 & - & $\begin{array}{c}\text { Sediment } \\
\text { (Ekman-Birge) }\end{array}$ \\
\hline
\end{tabular}

* Although some works are important references on Chironomidae in reservoirs of the São Paulo State (e.g. Strixino 1971, Rocha 1972, Strixino \& Strixino 1980, Valenti \& Froehlich 1986, Beyruth 1989, Brandimarte 1991) we have not cited them on the tables, because they had not showed their lists of morphospecies or the fauna was identified to the family or subfamily level . 
Table 2. List of Chironomidae recorded from lentic environments in the State of São Paulo (Brazil).* It is important to note that this list shows the names considered by each author and it does not present only currently taxonomic valid names.

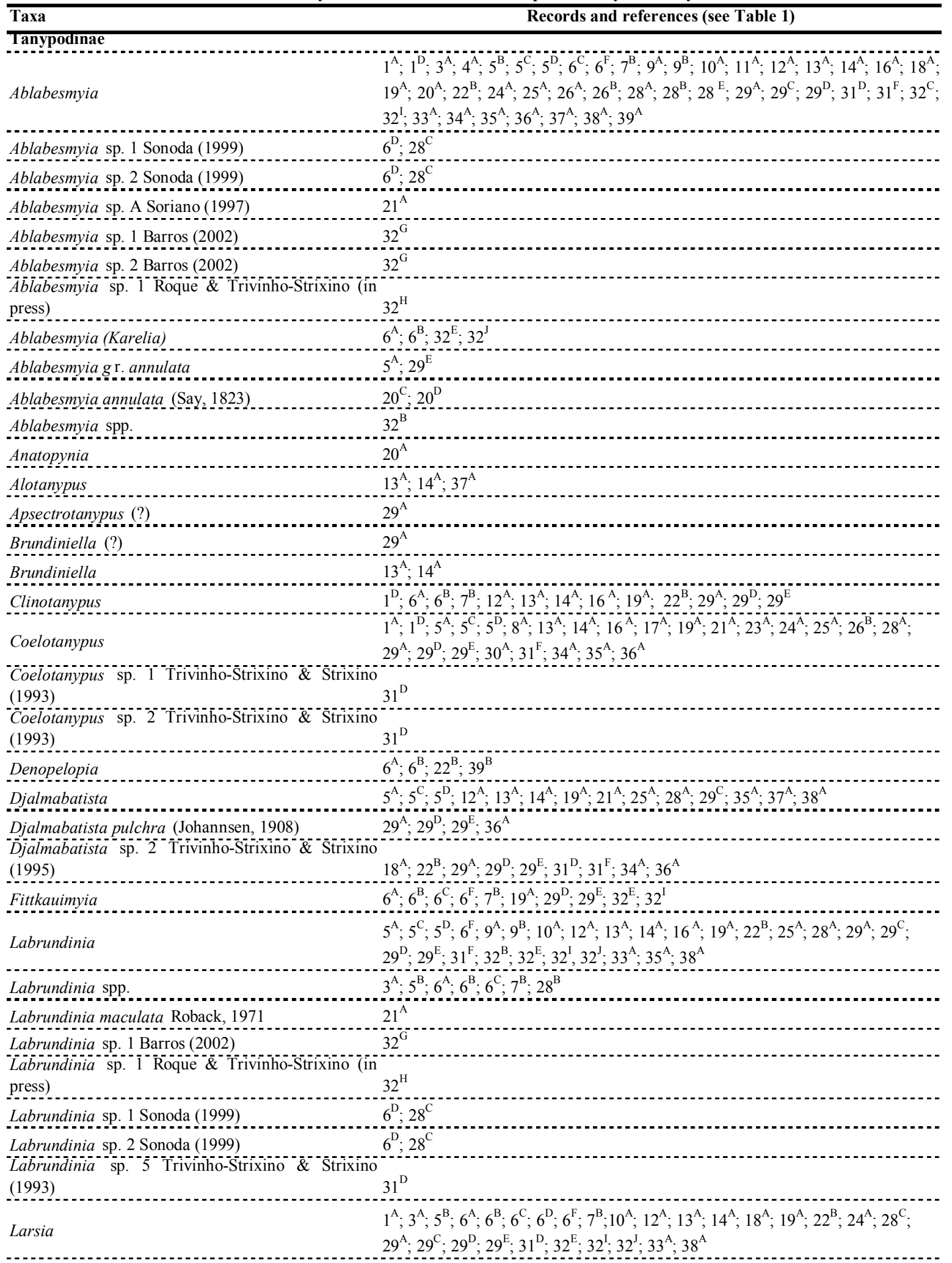




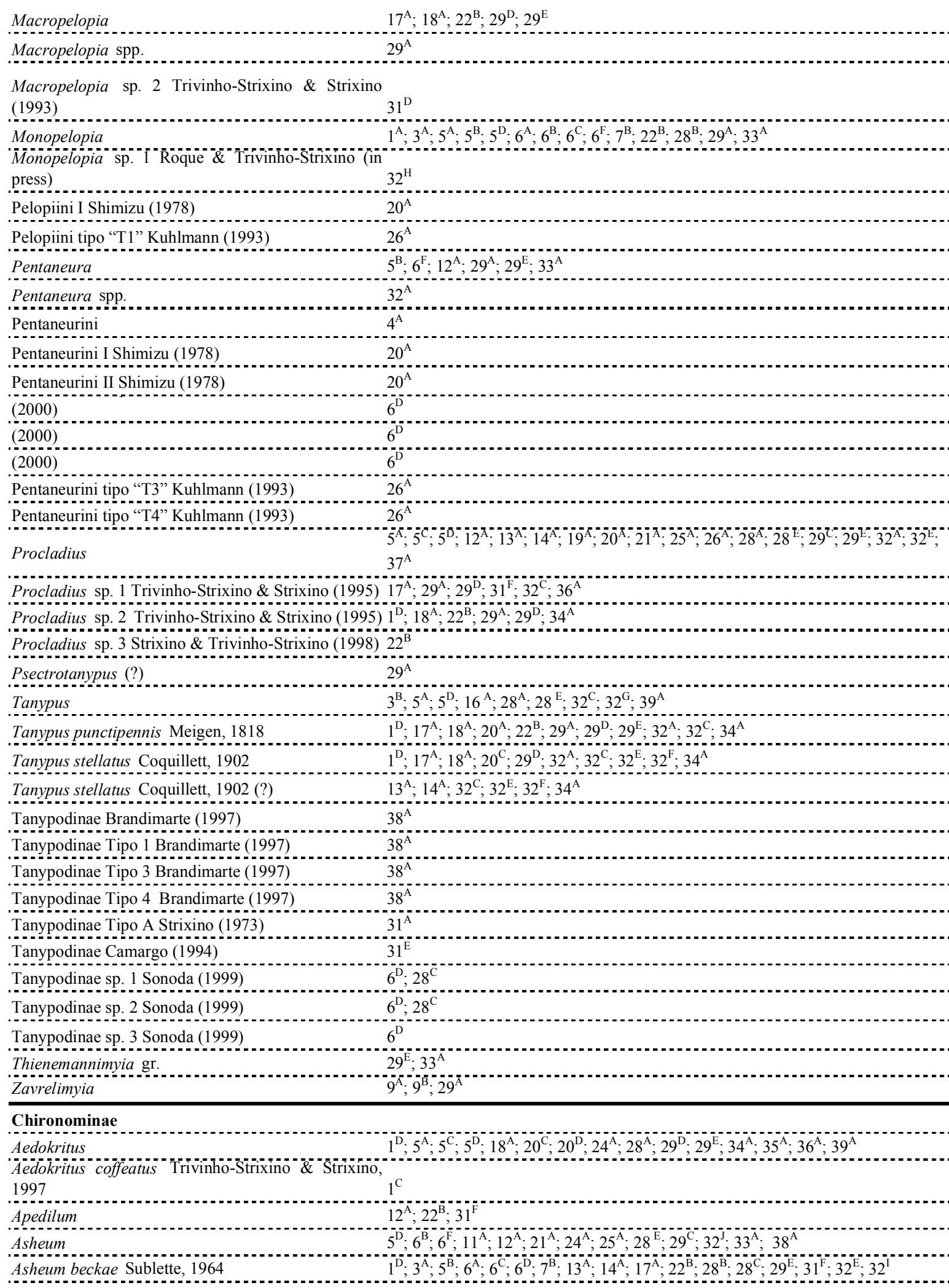




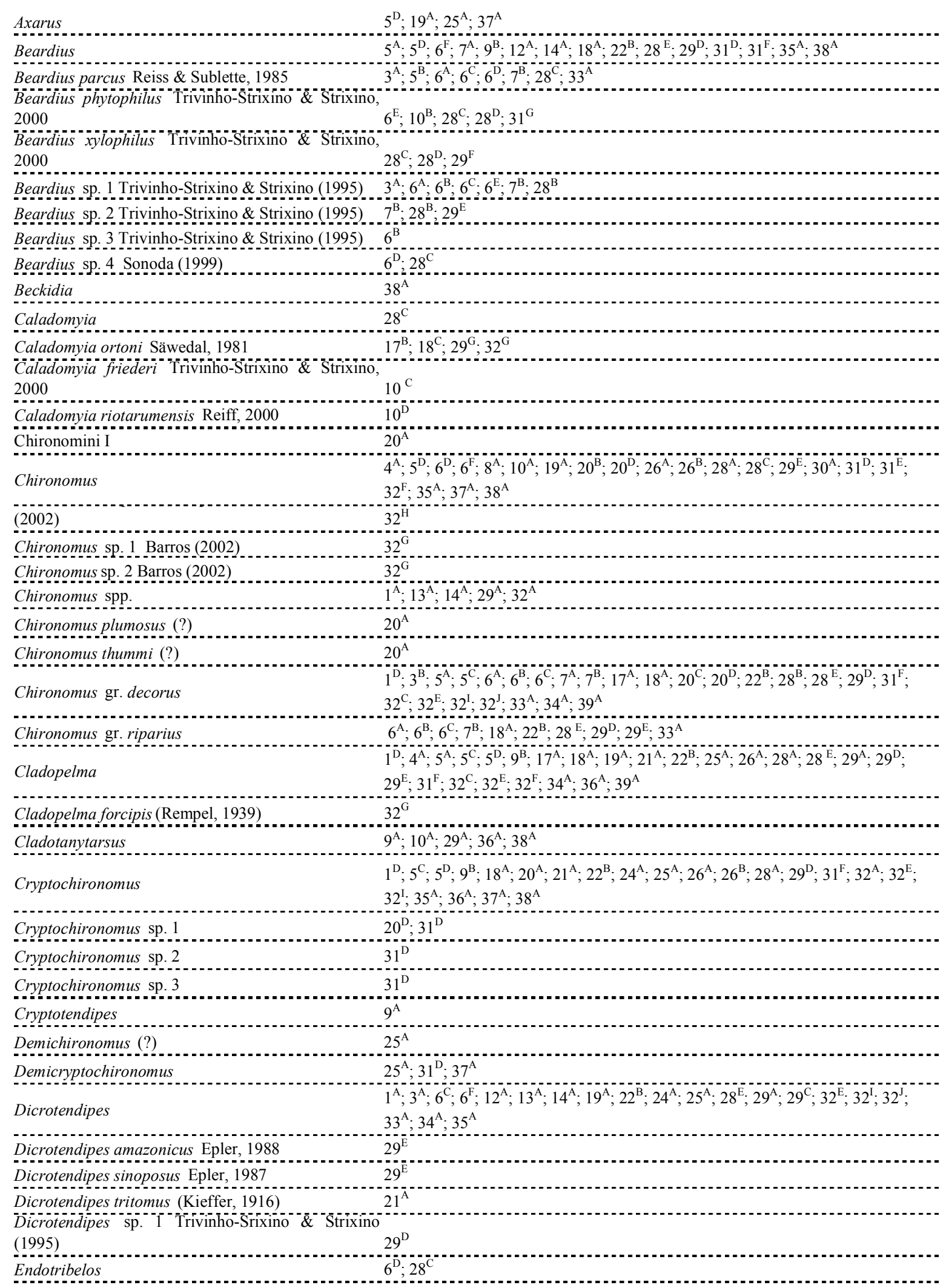

http://www.biotaneotropica.org.br 


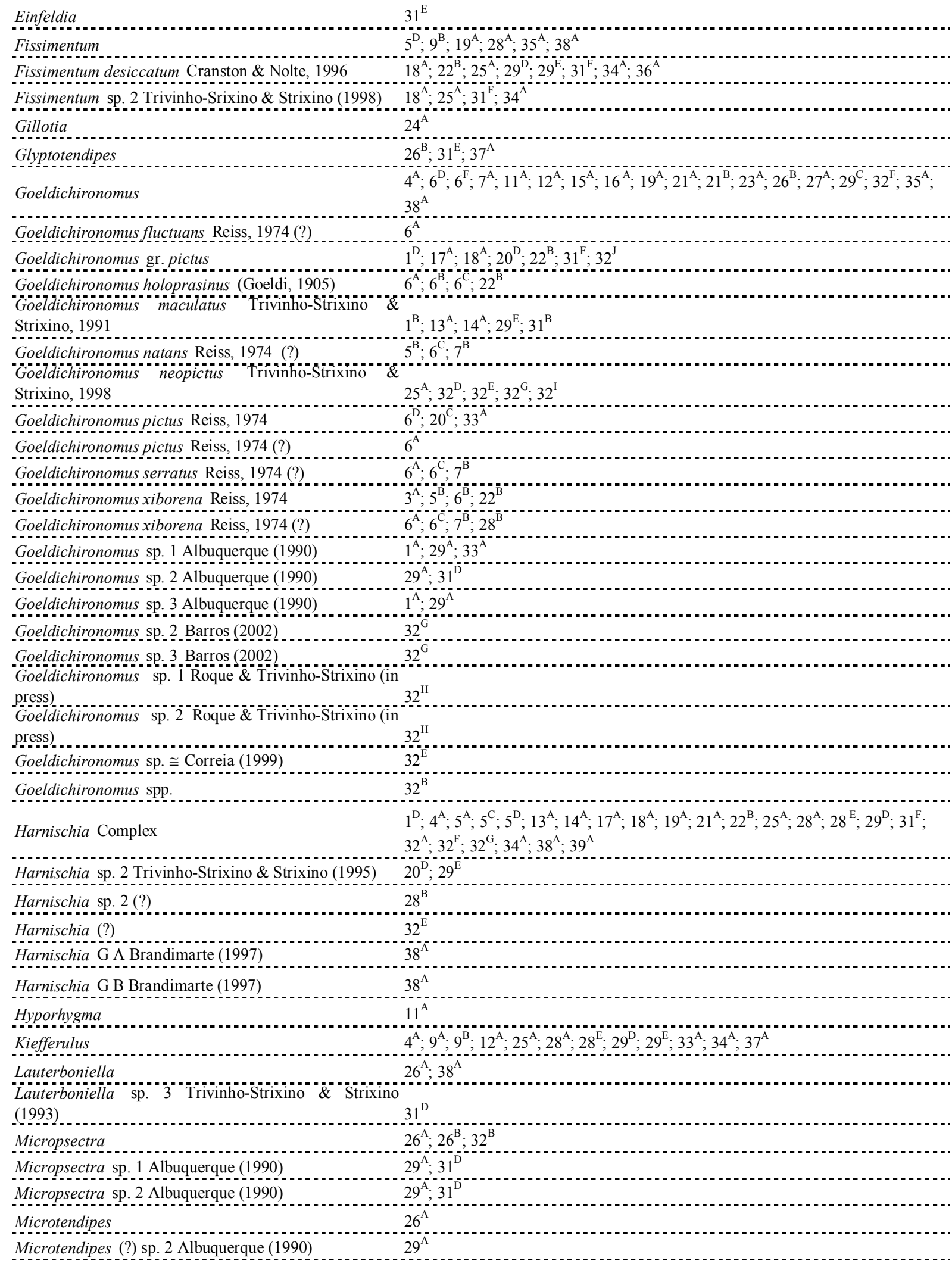




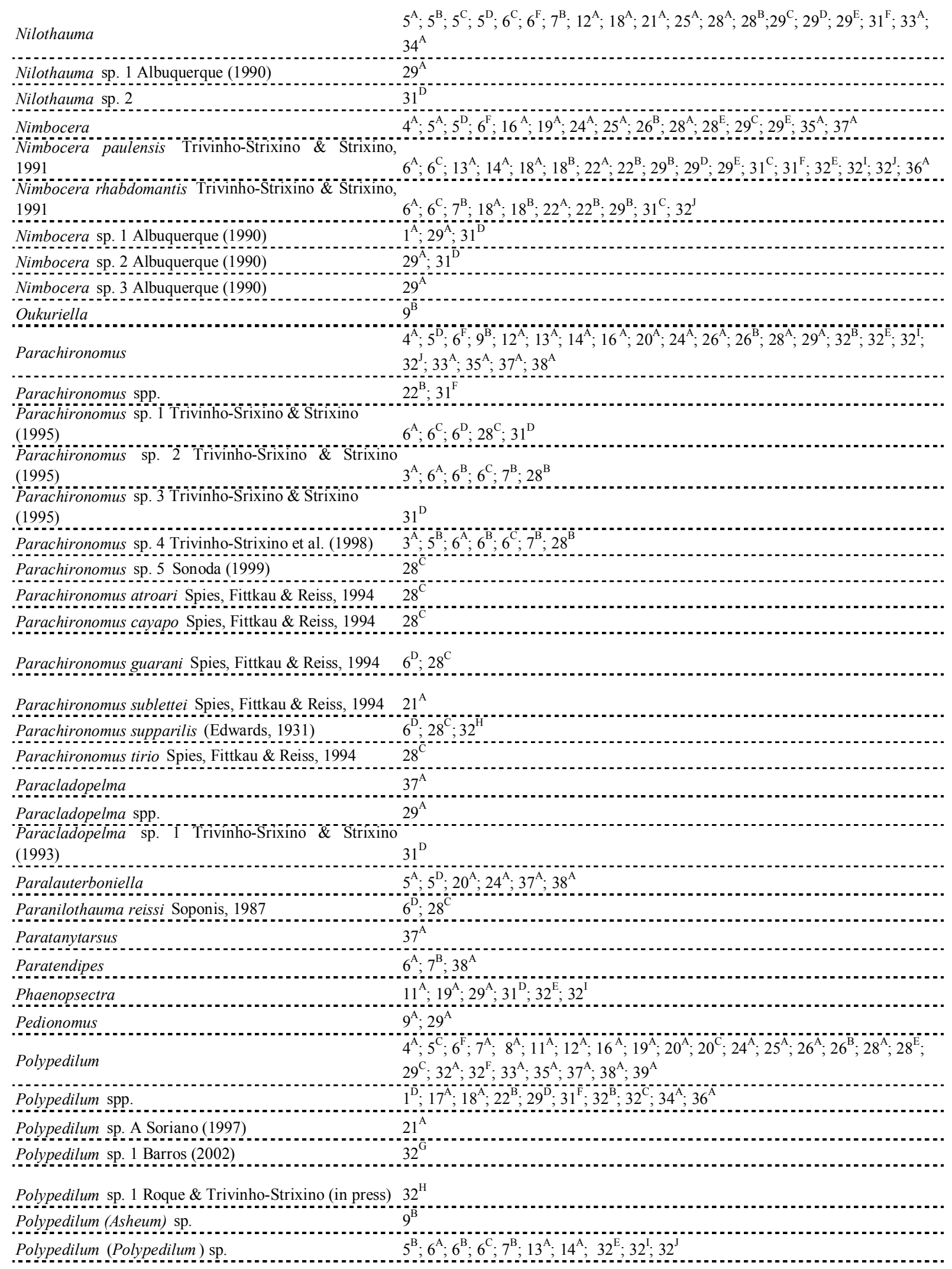




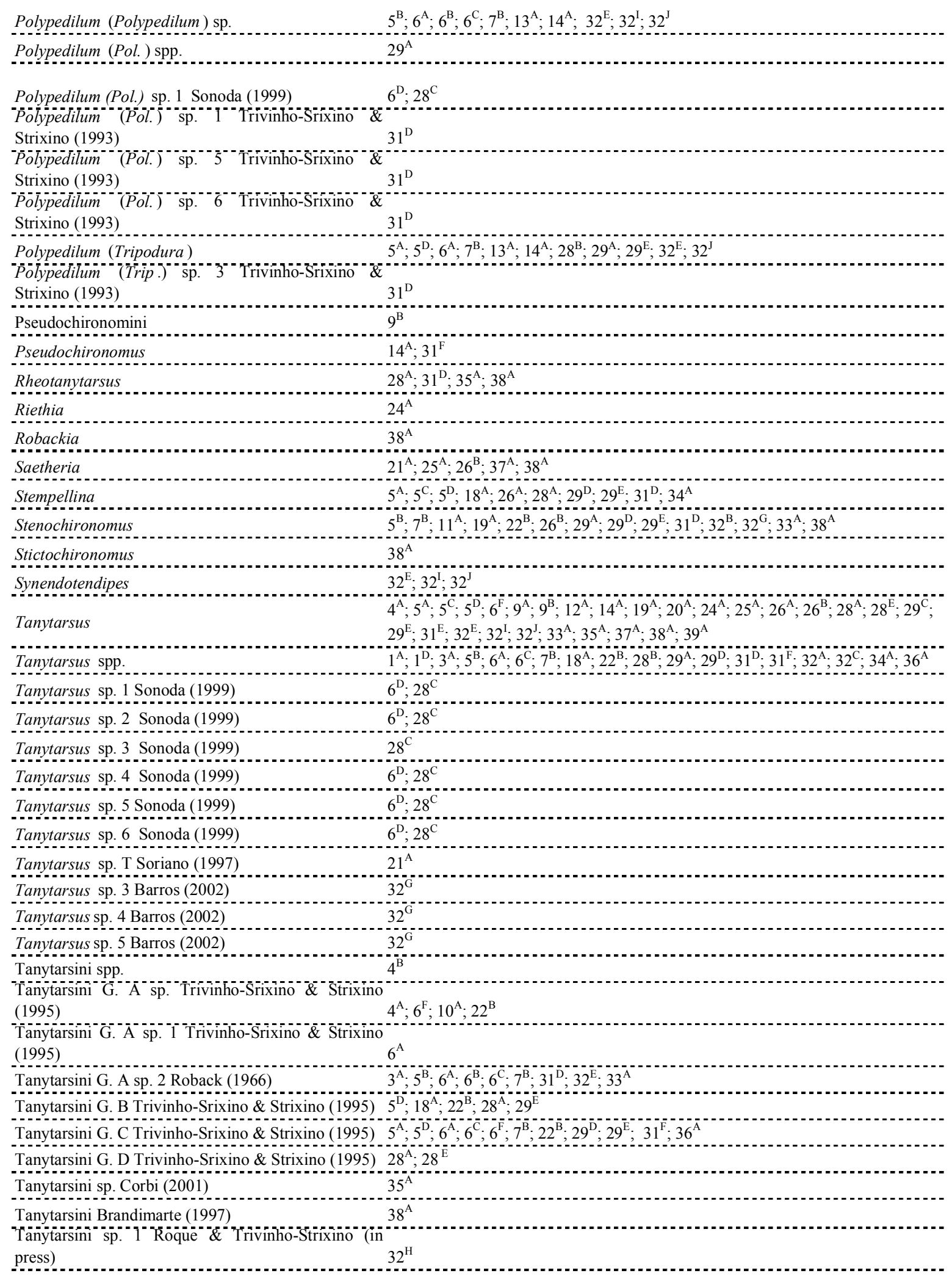


Tanytarsini sp. 2 Roque \& Trivinho-Strixino (in press) $32^{\mathrm{H}}$ Tanytarsini sp. ${ }^{3}$ Roque ${ }^{-}$Trivinhö-Strixino (in press) $32^{\mathrm{H}}$

Tribelos $32^{\mathrm{H}}$

Zavrelia $2^{\mathrm{A}} ; 5^{\mathrm{A}} ; 5^{\mathrm{B}} ; 5^{\mathrm{D}} ; 6^{\mathrm{C}} ; 6^{\mathrm{F}} ; 7^{\mathrm{B}} ; 11^{\mathrm{A}} ; 28^{\mathrm{B}} ; 31^{\mathrm{D}} ; 31^{\mathrm{E}} ; 33^{\mathrm{A}}$

Zavreliella $9^{\mathrm{A}}$

Xenochironomus $5^{\mathrm{D}} ; 17^{\mathrm{A}} ; 18^{\mathrm{A}} ; 19^{\mathrm{A}} ; 20^{\mathrm{D}} ; 22^{\mathrm{B}} ; 25^{\mathrm{A}} ; 29^{\mathrm{D}} ; 29^{\mathrm{E}} ; 35^{\mathrm{A}}$

Xestochironomus $9^{\mathrm{A}} ; 9^{\mathrm{B}} ; 24^{\mathrm{A}} ; 26^{\mathrm{A}} ; 33^{\mathrm{A}}$

Xestochironomus $38^{A}$

Chironomidae "X" Kuhlmann (1993) $26^{\mathrm{A}}$

Chironominae Tipo B Strixino (1973)

Chironominae Tipo C Strixino (1973)

Chironominae Tipo D Strixino (1973) $31^{\mathrm{A}}$

Chironominae Tipo E Strixino (1973)

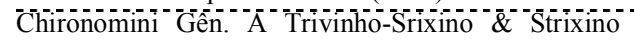

(1995)

Chironomini G. A Soriano (1997)

$28^{\mathrm{E}}$

Chironomini G. A sp. 4 Trivinho-Srixino \& Strixino

(1995) $\quad 5^{\mathrm{A}} ; 29^{\mathrm{A}}$

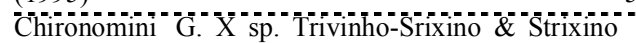

(1995) $5^{\mathrm{D}} ; 22^{\mathrm{B}}$

Chironomini A Santos \& Henry (2001)

Chironomini A Melão \& Rocha (1996)

Chironomini B Melão \& Rocha (1996)

Chironomini Brandimarte (1997)

Chironomini Tipo “T” Kuhlmann (1993) $26^{\mathrm{A}}$

\section{Orthocladiinae}

Corynoneura $3^{\mathrm{A}} ; 5^{\mathrm{B}} ; 6^{\mathrm{A}} ; 6^{\mathrm{C}} ; 6^{\mathrm{F}} ; 7^{\mathrm{B}} ; 9^{\mathrm{A}} ; 9^{\mathrm{B}} ; 19^{\mathrm{A}} ; 24^{\mathrm{A}} ; 28^{\mathrm{B}} ; 29^{\mathrm{A}} ; 33^{\mathrm{A}} ; 38^{\mathrm{A}}$

(1993)

Corynoneura sp. 2 Trivinho-Srixino \& Strixino

$(1993)$

$\begin{aligned} \text { Cricotopus } & 2^{\mathrm{A}} ; 3^{\mathrm{A}} ; 5^{\mathrm{A}} ; 5^{\mathrm{B}} ; 5^{\mathrm{D}} ; 6^{\mathrm{A}} ; 6^{\mathrm{C}} ; 6^{\mathrm{F}} ; 7^{\mathrm{B}} ; 9^{\mathrm{A}} ; 9^{\mathrm{B}} ; 11^{\mathrm{A}} ; 14^{\mathrm{A}} ; 24^{\mathrm{A}} ; 28^{\mathrm{B}} ; 28^{\mathrm{E}} ; 29^{\mathrm{A}} ; 29^{\mathrm{C}} ; 29^{\mathrm{E}} ; \\ & 31^{\mathrm{F}} ; 32^{\mathrm{B}} ; 32^{\mathrm{E}} ; 32^{\mathrm{H}} ; 32^{\mathrm{I}} ; 32^{\mathrm{J}} ; 33^{\mathrm{A}} ; 35^{\mathrm{A}} ; 38^{\mathrm{A}}\end{aligned}$

Cricotopus sp. 1 Trivinho-Srixino \& Strixino $(1993) 31$

Cricotopus sp. 2 Trivinho-Srixino \& Strixino (1993) $31^{\mathrm{D}}$

Eukiefferiella (?)

Metriocnemus

Nanocladius

Parametriocnemus

Psectrocladius

Thienemanniella

Thienemanniella sp. 1 Trivinho-Srixino \& Strixino

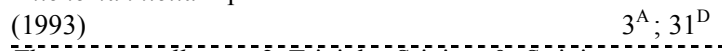

Thienemanniella sp. 2 Trivinho-Srixino \& Strixino

(1993) $31^{\mathrm{D}}$

Parakiefferiella

Orthocladiinae Brandimarte (1997)

Orthocladiini sp. 1 Sonoda (1999) $\quad 66^{\mathrm{D}} ; 28 \mathrm{C}$

Orthocladiini sp. 2 Sonoda (1999) 28

Observations about the table:

\section{Observations about the table:}

Species-level entries cite the author(s) and date of the first publication. With formally named species the format is "author, year" or "(author, year)", with morphospecies etc. it is "author (year)". In genus- and higher-level entries authors' names and dates are omitted to save space.

? - The respective author was not absolutely certain of the determination. 


\section{References}

AFONSO, A.A.O. 2002. Relações da fauna associada à Eichhornia azurea (Swartz) Kunth com as variáveis abióticas em lagoas laterais de diferentes graus de conexão ao Rio Paranapanema (zona de desembocadura na Represa de Jurumirim, SP). Tese de doutorado, Universidade Estadual Paulista, Botucatu.

ALBUQUERQUE, L.B. 1990. Entomofauna aquática do litoral de dois reservatórios da região de São Carlos. Dissertação de mestrado, Universidade Federal de São Carlos, São Carlos.

ALVES, R.G. \& STRIXINO, G. 1997. Chironomidae e Oligochaeta de uma lagoa da planicie de inundação do Rio Mogi-Guaçu: Lagoa do Diogo (Estação Ecológica do Jataí, Luiz Antônio, SP). In Anais do VIII Seminário Regional de Ecologia. PPG-ERN, São Carlos, p.141-149.

ALVES, R.G. \& STRIXINO, G. 2000. Influência da variação do nível da água sobre a comunidade macrobentônica da Lagoa do Diogo (Luiz Antônio, SP). In Estação Ecológica de Jataí: estudos integrados em ecossistemas (J.E. Santos \& J.S.R. Pires, ed). Rima, São Carlos, p.733742

ALVES, R.G. 1998. Dinâmica espaço-temporal dos macroinvertebrados bentônicos de uma lagoa marginal de drenagem. Tese de doutorado, Universidade Federal de São Carlos, São Carlos.

ANAYA, M, FAGUNDES, R.C., KUHLMANN, M.L., SHIMIZU, G.Y., TAKEDA, A.M., CASTILHO, M.S.M., KUBO, E., LEMOS, M.M.G. \& SILVA, C.P.D. 1997. Variação batimétrica na estrutura da comunidade bentônica na represa de Paraibuna (SP). In Anais do VIII Seminário Regional de Ecologia. PPG-ERN, São Carlos, p.99-111.

ARMITAGE, P.D., CRANSTON, P.S. \& PINDER, L.C.V. 1995. The Chironomidae: the biology and ecology of non-biting midges. Chapman \& Hall, London.

BARROS, T. S. 2002. Exúvias de pupas de Chironomidae (Diptera) na caraterização de corpos d'água do campus e arredores da Universidade Federal de São Carlos. Relatório Parcial de Iniciação Científica, Universidade Federal de São Carlos, São Carlos.

BEYRUTH, Z. 1989. Estudo ecológico sanitário de um lago marginal ao Rio Embu-Mirim - Itapecerica da Serra, São Paulo e ensaio sobre a possibilidade de utilização da macrófita Eichhornia crassipes (Mart.) Solms como indicadora de poluição. Dissertação de mestrado, Universidade de São Paulo, São Paulo.

BORGHOFF-GONÇALVES, C.\& TRIVINHO-STRIXINO, S. 1997. Macroinvertebrados bentônicos em tanques de piscicultura. In Anais do VIII Seminário Regional de Ecologia. PPG-ERN, São Carlos, p.43-52.
BRANDIMARTE,A.L. 1991. Macrofauna bentônica da zona litoral da Represa de Paraibuna (SP): a influência de diferentes ecossistemas terrestres perimetrais. São Paulo. Dissertação de mestrado, Universidade de São Paulo, São Paulo.

BRANDIMARTE, A.L. 1997. Impactos limnológicos da construção do reservatório de aproveitamento múltiplo do Rio Mogi-Guaçu (SP, Brasil) sobre a comunidade de invertebrados bentônicos. Tese de doutorado, Universidade de São Paulo, São Paulo.

CAMARGO, A.F.M. 1984. Aspectos ecológicos de três espécies de macrófitas aquáticas da Represa do Lobo, SP: Interações com macroinvertebrados e decomposição. Dissertação de Mestrado, Universidade Federal de São Carlos, São Carlos.

CAMPOS, A.F. 1999. Macroinvertebrados bentônicos em viveiros de criação de peixes do Centro Nacional de Pesquisa de Peixes Tropicais- CEPTA, Pirassununga. Dissertação de mestrado, Universidade Federal de São Carlos, São Carlos.

COFFMAN, W.P. 1995. Conclusions. In The Chironomidae: the biology and ecology of non-biting midges (P.D. Armitage, P.S. Cranston, L.C.V Pinder, eds.). Chapman \& Hall, London, p.436-447.

CORBI, J.J. \& TRIVINHO-STRIXINO, S. 2002. Spatial and bathymetric distribution of the macrobenthic fauna of the Ribeirão das Anhumas reservoir (Américo brasiliense-SP, Brazil). Acta limnol. Bras. 14(1): 35-42.

CORBI, J.J. 2001. Distribuição espacial e batimétrica dos macroinvertebrados bentônicos da represa do Ribeirão das Anhumas (Américo Brasiliense - SP). Dissertação de Mestrado, Universidade Federal de São Carlos, São Carlos.

CORREIA, L.C.S. \& TRIVINHO-STRIXINO, S. 1998. Macroinvertebrados da rizosfera de Scirpus cubensis na Lagoa do Infernão (Estação Ecológica de Jataí - SP): estrutura e função. Acta limnol. Bras. 10(1): 37-47.

CORREIA, L.C.S. \& TRIVINHO-STRIXINO, S. Chironomidae (Diptera) em substratos artificiais num pequeno reservatório: Represa do Monjolinho (São Carlos, SP). Entomolologia y Vectores., submitted a.

CORREIA, L.C.S. \& TRIVINHO-STRIXINO, S. Colonization of two different macrophyte debris by Chironomidae and other macroinvertebrates in an artificial lake (São PauloBrazil), Mem Inst Oswaldo Cruz. submitted b.

CORREIA, L.C.S. 1996. Estrutura e função da fitofauna associada à rizosfera de Scirpus cubensis na lagoa do Infernão (Reserva Ecológica de Jataí - SP). Monografia de graduação, Universidade Federal de São Carlos, São Carlos. 
CORREIA, L.C.S. 1999. Comparação entre as assembléias de macroinvertebrados associadas a substratos naturais e artificiais na Represa do Monjolinho (São Carlos- SP). Dissertação de Mestrado, Universidade Federal de São Carlos, São Carlos.

CRANSTON, P.S. 1995. Medical significance. In The Chironomidae: the biology and ecology of non-biting midges (P.D. Armitage, P.S. Cranston, L.C.V Pinder, eds.). Chapman \& Hall, London, p.365-384.

DORNFELD, C.B. 1999. Comunidade de macroinvertebrados fitófilos associados a Salvinia sp. e Myriophyllum sp. num reservatório no Parque Ecológico Dr. Antônio Teixeira Vianna, São Carlos, SP. Monografia de graduação, Universidade Federal de São Carlos, São Carlos.

DORNFELD, C.B. 2002. Utilização de análises limnológicas, bioensaios de toxicidade e macroinvertebrados bentônicos para o diagnóstico ambiental do reservatório de Salto Grande (Americana, SP). Dissertação de mestrado, Universidade de São Paulo, São Carlos.

EPLER, J.H. 2001. Identification Manual for the larval Chironomidae (Diptera) of North and South Carolina. A guide to the taxonomy of the midges of the southeastern United States, including Florida. Special Publication SJ2001-SP13. North Carolina Department of Environment and Natural Resources, Raleigh, NC, and St. Johns River Water Management District, Palatka, FL. 526 pp. http:// home.earthlink.net/ johnepler/index.html

FERRARESE, U. 1993. Chironomids of Italian rice fields. Netherlands Journal of Aquatic Ecology. 26: 341-346.

FITTKAU, E.J. 2001. Amazonian Chironomidae (Diptera, Chironomidae): a contribution to chironomid research in the Neotropics. Amazoniana. XVI (3/4): 313-323.

KUHLMANN, M.L. 1993. Estudo da comunidade de invertebrados bentônicos da zona profunda da Represa de Paraíbuna (SP). Dissertação de Mestrado, Universidade de São Paulo, São Paulo.

KUHLMANN, M.L., TRUZZI, A C., JOHNSCHERFORNASARO, G. \& LAMPARELLI, M.C. 1997. A fauna bentônica do Complexo Billings (SP). Relatório Técnico, CETESB, São Paulo.

LÉO, F.C. 1999. Avaliação ambiental da represa do Monjolinho (Campus UFSCar) através dos macroinvertebrados bentônicos. Monografia de graduação, Universidade Federal de São Carlos, São Carlos.

LEWINSOHN, T.L. \& PRADO, P.I. 2003. Biodiversidade Brasileira: síntese do estado atual do conhecimento. Contexto, São Paulo.

MELÃO, M.G.G. \& ROCHA, O. 1996. Macrofauna associada a Metania spinata (Carter, 1881), Porifera, Metaniidae. Acta Limnol. Bras. 8: 59-64.
MENDES, H.F. 2002. Rearing Tanypodinae, Telmatogetoninae and Orthocladiinae in Brazil - An empirical approach. Chironomus newsletter. 15: 29-32.

MESSIAS, M.C. 2000. Sebastião José de Oliveira, uma vida dedicada ao Instituto Oswaldo Cruz. Entomolologia y Vectores. 7(3): 239-253.

NEW, T.R. 1998. Invertebrate surveys for conservation. Oxford University Press, Oxford.

PAMPLIN, P.A.Z. 1999. Avaliação da qualidade ambiental da Represa de Americana (SP, Brasil) com ênfase no estudo da comunidade de macroinvertebrados bentônicos e parâmetros ecotoxicológicos. Dissertação de mestrado, Universidade de São Paulo, São Paulo.

PAMPLIN, P.A.Z. 2002. Estudo comparativo da estrutura da comunidade bentônica de duas represas com diferenças no grau de eutrofização. Relatório FAPESP, Universidade Federal de São Carlos, São Carlos.

ROCHA, A.A. 1972. Estudo sobre a fauna bentônica da represa de Americana no Estado de São Paulo, Instituto de Biociências, USP. Tese de doutorado, Universidade de São Paulo, São Paulo.

RODRIGUES, M.H. 1997. Estudo da fauna de Chironomidae (Diptera) do sedimento na Represa do Beija Flor, na Estação Ecológica do Jataí, Luiz Antônio, SP. Dissertação de Mestrado, Universidade Federal de São Carlos, São Carlos.

ROQUE, F.O. \& TRIVINHO-STRIXINO, S. Enxameamento em Chironomidae (Diptera) na represa Monjolinho, São Carlos - SP. Entomologia Y Vectores, submitted.

ROQUE, F.O. 1999. Macroinvertebrados aquáticos do Parque Estadual do Jaraguá. Relatório Técnico, Projeto BIOTA-Jaraguá, São Paulo.

ROSENBERG, D.M. 1992. Freshwater biomonitoring and Chironomidae. Netherlands Journal of Aquatic Ecology. 26(2-4): 101-122.

SANTOS, C.M. \& HENRY, R. 2001. Composição, distribuição e abundância de Chironomidae (Diptera- Insecta) na Represa de Jurumirim (Rio Paranapanema- SP). Acta Limnol. Bras. 23(2): 99-115.

SANTOS, C.M. 2002. Distribuição de mesoinvertebrados em um lago eutrofizado (Lago das Garças- SP) e excreção de fosfato e amônio por Chironomidae (Diptera). Tese de doutorado, Universidade Estadual Paulista, Botucatu.

SHIMIZU, G.Y. 1978. Represa de Americana: aspectos do bentos litoral. Dissertação de mestrado, Universidade Federal de São Paulo, São Paulo.

SHIMIZU, G.Y. 1981. Represa de Americana: um estudo da distribuição batimétrica da fauna bentônica. Tese de doutorado, Universidade Federal de São Paulo, São Paulo. 
SONODA, K.C.\& TRIVINHO-STRIXINO, S. 2000. Dinâmica de emergência de Chironomidae (Diptera) da fitofauna de Cabomba piauhyensis Gardney, 1844, na Lagoa do Infernão (Estação Ecológica de Jataí, Luiz Antônio, SP). In Estação Ecológica de Jataí: estudos integrados em ecossistemas (J.E. Santos \& J.S.R. Pires, ed). Rima, São Carlos, p.743-754.

SONODA, K.C. 1999. Chironomidae (Diptera) da fitofauna de Cabomba piauhyensis Gardney 1844. Dissertação de Mestrado, Universidade Federal de São Carlos, São Carlos.

SORIANO, A.J.S. 1997. Distribuição espacial e temporal de invertebrados bentônicos da represa de Barra Bonita (SP). Dissertação de Mestrado, Universidade Federal de São Carlos, São Carlos.

SPIES, M. \& REISS, F. 1996. Catalog and bibliography of Neotropical and Mexican Chironomidae. Spixiana Suppl. 22:61-119.

STRIPARI, N.L. \& HENRY, R. 2002. The invertebrate colonization during decomposition of Eichhornia azurea Kunth in a lateral lake in the mouth zone of Paranapanema River into Jurumirim Reservoir (São Paulo, Brazil). Braz. J. Biol. 2:293-310.

STRIXINO, G. \& STRIXINO, S.T. 1980. Macroinvertebrados do fundo da Represa do Lobo (Estado de São Paulo Brazil). I. Distribuição e abundância de Chironomidae e Chaoboridae (Diptera). Tropical Ecology. 21(1): 16-23.

STRIXINO, G. \& STRIXINO, S.T. 1982. Macrobentos da represa do Monjolinho (São Carlos - SP). Rev. Brasil. Biol. 42 (1): 165-170.

STRIXINO, G. \& STRIXINO, S.T. 1984. Macroinvertebrados associados a tapetes flutuantes de Eichornia crassipes (Mart.) Solms, de um reservatório. In Anais do IV Seminário Regional de Ecologia. PPG-ERN, São Carlos, p.375-397.

STRIXINO, G. \& TRIVINHO-STRIXINO, S. 1998. Povoamentos de Chironomidae (Diptera) em lagos artificiais. In Ecologia de Insetos Aquáticos (J.L. Nessimian \& A.L. Carvalho, eds). Séries Oecologia Braziliensis, PPG-UFRJ, Rio de Janeiro. v.1, p.141-154.

STRIXINO, G., TRIVINHO-STRIXINO, S. \& ALVES, R.G. 1997. Macroinvertebrados bentônicos de lagoas marginais da planície de inundação do Rio Mogi-Guaçu (Estação Ecológica de Jataí). In Anais do VIII Seminário Regional de Ecologia. PPG-ERN, São Carlos, p.33-42.

STRIXINO, G.M.A. 1971. Ensaio para um estudo ecológico da macrofauna do fundo da Represa de Americana (SP). Dissertação de mestrado, Universidade de São Paulo, São Paulo.

STRIXINO, G.M.A. 1973. Sobre a ecologia dos macroinvertebrados do fundo, na Represa do Lobo. Tese de doutorado, Universidade de São Paulo, São Paulo.
TRIVINHO-STRIXINO, S. 1997. Nova espécie do gênero Aedokritus Roback, 1958 (Diptera, Chironomidae), com descrição das formas imaturas. Revta. Bras. Ent. 41(1): 13-16.

TRIVINHO-STRIXINO, S. \& SANSEVERINO,A.M. 2003. Tanytarsus rhabdomantis: New combination for Nimbocera rhabdomantis Trivinho-Strixino \& Strixino, 1991 (Diptera, Chironomidae). Zootaxa 389: 1-10.

TRIVINHO-STRIXINO, S. \& STRIXINO, G. 1991a. Nova espécie de Goeldichironomus Fittkau (Diptera, Chironomidae) do Brasil. Revta. Bras. Ent. 35(3): 593602.

TRIVINHO-STRIXINO, S. \& STRIXINO, G. 1991b. Duas novas espécies de Nimbocera Reiss (Diptera, Chironomidae) do Estado de São Paulo, Brasil. Revta. bras. Ent. 35(1): 173-178.

TRIVINHO-STRIXINO, S. \& STRIXINO, G. 1993. Estrutura da comunidade de insetos aquáticos associados à Pontederia lanceolata Nuttal. Rev. Brasil. Biol. 53(1): 103-111.

TRIVINHO-STRIXINO, S. \& STRIXINO, G. 1995. Larvas de Chironomidae (Diptera) do Estado de São Paulo: Guia de Identificação e diagnose de gêneros. PPG-ERN/ UFSCar, São Carlos.

TRIVINHO-STRIXINO, S. \& STRIXINO, G. 1998a. Chironomidae (Diptera) associados a troncos de árvores submersos. Revta. bras. Ent. 41(2-4): 173-178.

TRIVINHO-STRIXINO, S. \& STRIXINO, G. 1998b. Goeldichironomus neopictus, a new species from the southeast of Brazil: description and bionomic information. Spixiana. 21(3): 271-278.

TRIVINHO-STRIXINO, S. \& STRIXINO, G. 1999. Insetos dípteros: quironomídeos. In Biodiversidade do Estado de São Paulo: Invertebrados de água doce (Ismael et al., eds.). FAPESP, São Paulo, p.141-148.

TRIVINHO-STRIXINO, S. \& STRIXINO, G. 2000a. Two new species of Beardius Reiss et Sublette, 1985 (Diptera, Chironomidae) from Southeastern Brazil. In Late 20th Century Research on Chironomidae: an Anthology from the 13th International Symposium on Chironomidae (O. Hoffrichter, ed). Aachen: Shaker, Freiburg, p.245-250.

TRIVINHO-STRIXINO, S. \& STRIXINO, G. 2000b. Anew species of Caladomyia Säwedal, 1981, with description of the female and immature stages. Spixiana 23 (2): 167-173.

TRIVINHO-STRIXINO, S. \& STRIXINO, G. 2003. The immature stages of two Caladomyia Säwedall, 1981 species, from São Paulo State, Brazil (Chironomidae, Chironominae, Tanytarsini). Revta. Bras. Ent. 47(4): $597-$ 602. 
TRIVINHO-STRIXINO, S., CORREIA, L.C.S. \& SONODA, K. 2000. Phytophilous Chironomidae (Diptera) and other macroinvertebrates in the ox-bow Infernão Lake (Jataí Ecological Station, Luiz Antônio, SP, Brazil). Rev. Brasil. Biol. 60(3): 527-535.

TRIVINHO-STRIXINO, S., GESSNER, F.A. \& CORREIA, L.C.S. 1998. Macroinvertebrados associados a macrófitas aquáticas das lagoas marginais da Estação Ecológica de Jataí (Luiz Antônio -SP). In Anais do VIII Seminário Regional de Ecologia. PPG-ERN, São Carlos, p.1189-1198.

VALENTI, W.C. \& FROEHLICH, O. 1986. Estudo da diversidade da taxocenose de Chironomidae dez Reservatórios do Estado de São Paulo. Ciência e Cultura. 38(4): 703-707.

WALKER, I.R. 1995. Chironomids as indicators of past environmental change. In The Chironomidae: the biology and ecology of non-biting midges (P.D. Armitage, P.S. Cranston \& L.C.V. Pinder, eds). Chapman \& Hall, London, p.405-422.

Title: A Review Of Chironomidae Studies In Lentic Systems In The State Of São Paulo, Brazil

Authors: Fábio de Oliveira Roque; Leny Célia da Silva Correia;Susana Trivinho-Strixino; Giovanni Strixino

Biota Neotropica, Vol. 4( number 2): 2004

http://www.biotaneotropica.org.br/v4n2/pt/ abstract?short-communication+BN03104022004

Date Received 05/20/2003

Revised 07/19/2003

Accepted 08/01/2003

ISSN 1676-0611

http://www.biotaneotropica.org.br 